\title{
Advances in functional MRI of the human brain
}

\author{
J. Frahm*, P. Dechent, J. Baudewig, K.D. Merboldt \\ Biomedizinische NMR Forschungs GmbH, Max-Planck-Institut für Biophysikalische Chemie, D-37070 Göttingen, Germany
}

Received 13 October 2003

Contents

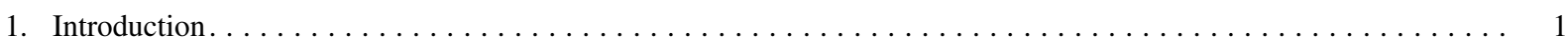

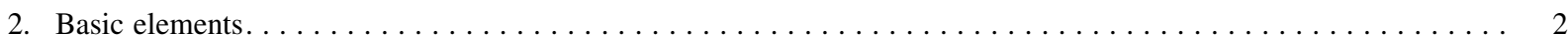

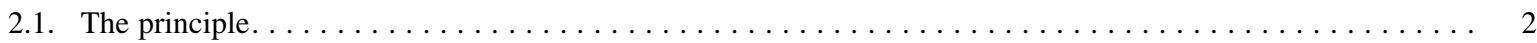

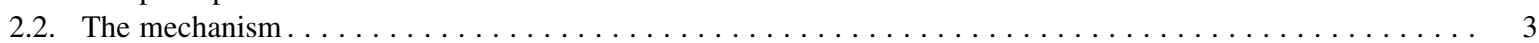

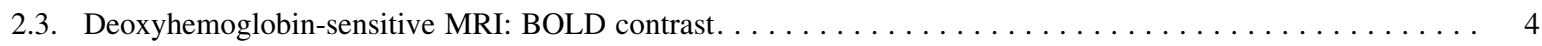

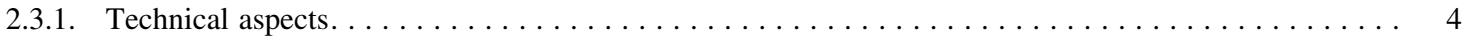

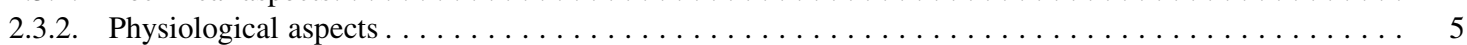

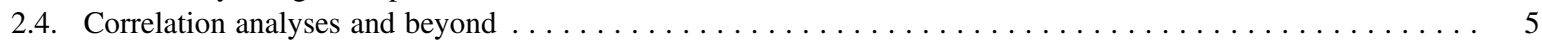

2.5. A typical experiment: retinotopic organization of the visual cortex $\ldots \ldots \ldots$

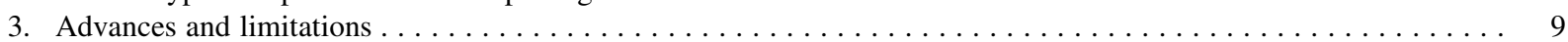

3.1. On the neural basis of BOLD MRI responses $\ldots \ldots \ldots \ldots \ldots$

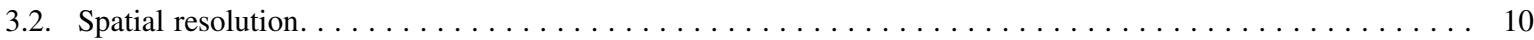

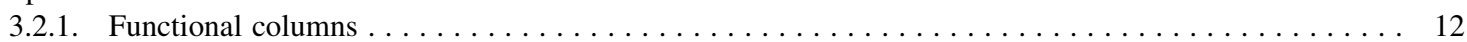

3.2.2. Filtering and multi-subject averaging $\ldots \ldots \ldots \ldots \ldots \ldots \ldots$

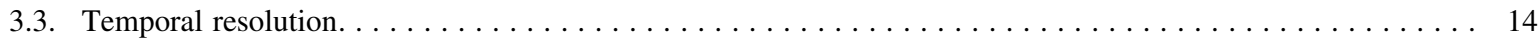

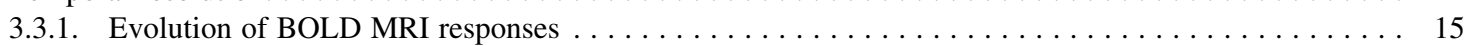

3.3.2. EEG-correlated mapping of single events: epilepsy $\ldots \ldots \ldots \ldots \ldots$

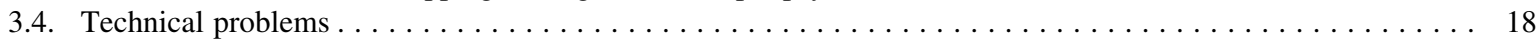

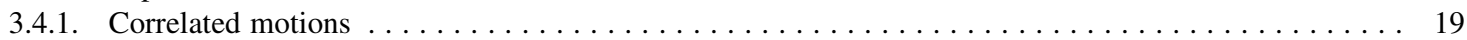

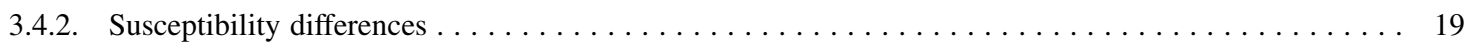

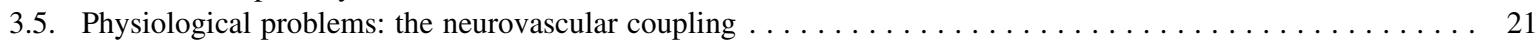

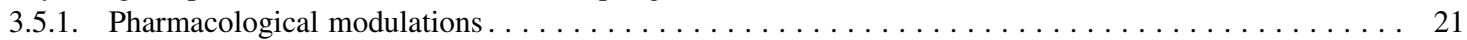

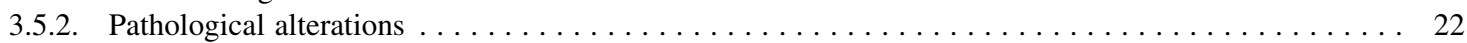

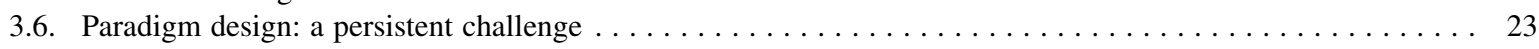

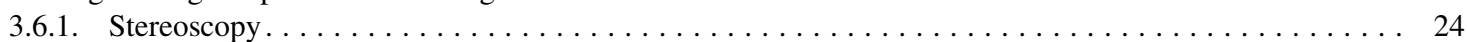

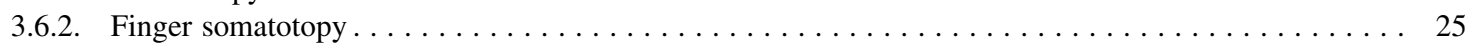

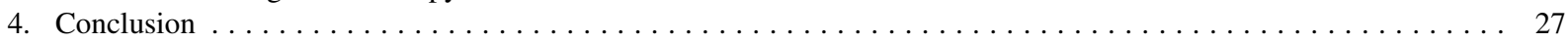

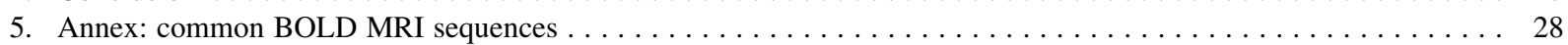

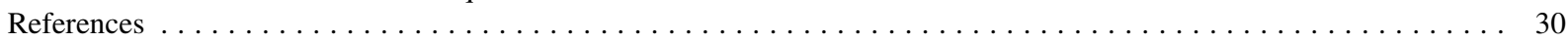

Keywords: Magnetic resonance imaging; Functional MRI; BOLD MRI; Human brain; Functional anatomy; Brain function

\section{Introduction}

After about a decade of methodological developments and promising applications in both basic and clinical

\footnotetext{
* Corresponding author. Tel.: +49-551-201-1721; fax: +49-551-2011307.

E-mail address: jfrahm@gwdg.de (J. Frahm).
}

neuroscience [1-7], this work summarizes the key elements in the experimental design and use of magnetic resonance imaging (MRI) for mapping brain function in humans. Based on an improved understanding of the underlying physiologic mechanisms and a growing number of applications to selected brain systems, the main purpose of this contribution is to present a discussion of the general potential and the specific challenges of this continuously 


$\begin{array}{lll}\text { Nomenclature } & \text { MEG } & \begin{array}{l}\text { magnetoencephalography } \\ \text { magnetic resonance imaging }\end{array} \\ \text { ATP adenosinetriphosphate } & \text { MRI } & \begin{array}{l}\text { TMS } \\ \text { transcranial magnetic stimulation }\end{array} \\ \text { AMT active motor threshold } & \text { M1 } & \text { primary motor cortex } \\ \text { BOLD blood-oxygenation-level-dependent } & \text { LPC } & \text { lateral premotor cortex } \\ \text { CNR contrast-to-noise ratio } & \text { RF } & \text { radiofrequency } \\ \text { CSF cerebrospinal fluid } & \text { S1 } & \text { primary sensory cortex } \\ \text { D1-D5 digit 1 (thumb)-digit 5 (little finger) } & \text { SFO } & \text { sequential finger-to-thumb opposition } \\ \text { EEG electroencephalography } & \text { SMA } & \text { supplementary motor area } \\ \text { EPI echo-planar imaging } & \text { SNR } & \text { signal-to-noise ratio } \\ \text { FLASH fast low angle shot } & \text { SPM } & \text { statistical parametric mapping } \\ \text { ICA internal carotid artery } & & \end{array}$

expanding field. Rather than providing a comprehensive survey, emphasis will be placed on both characteristic advantages that render MRI particularly attractive for functional neuroimaging and potential problems that may hamper the interpretation of the experimental results.

Apart from discussing crucial aspects ranging from cerebral hemodynamics to data acquisition and parametric mapping, specific points addressed are the neural basis of the functional MRI signal as well as the achievable spatial and temporal resolution. Technical complications will be discussed as well as limitations resulting from pharmacological and pathological modulations of the neurovascular coupling. A final section covers the increasingly difficult translation of a neuroscientific question into a proper MRI-compatible paradigm.

This contribution follows the mainstream of ideas in functional MRI by focusing on deoxyhemoglobin-sensitive MRI approaches which rely on blood-oxygenation-leveldependent (BOLD) contrast first discovered by Ogawa and colleagues in high-field MRI of rat brain [8]. The ease of use of the BOLD concept directly led to the first human applications in 1992 [9-12] and replaced an earlier proposal for functional MRI by Belliveau and colleagues [13] who measured activation-induced changes in cerebral blood volume after a bolus administration of a paramagnetic contrast agent. There are other approaches to functional brain mapping which are outside the scope of this article such as perfusion studies which aim at a direct detection of changes in cerebral blood flow using arterial spin labeling [14-16].

Functional neuroimaging studies of animals have recently been reviewed in this journal [17] and are therefore not included. When based on BOLD contrast, animal studies face specific problems due to the interaction of most anesthetics with the responsiveness of the cerebral vasculature (Section 3.5.1). On the other hand, such studies may exploit novel contrast agents not (yet) applicable to humans. A prominent example is the use of paramagnetic $\mathrm{Mn}^{2+}$ ions which shorten the $\mathrm{T} 1$ relaxation properties of affected water protons and thereby enhance the signal of $\mathrm{T} 1$-weighted MRI sequences [18-22]. In brain tissue $\mathrm{Mn}^{2+}$ ions behave as analogues to $\mathrm{Ca}^{2+}$ ions which-depending on brain function-are taken up by active neurons through voltage-gated calcium channels and axonally transported to respective cortical projection fields. Accordingly, the exposure of brain tissue to $\mathrm{Mn}^{2+}$ by systemic administration [22] or intracerebral injection bears the potential for unraveling neuroaxonal connectivities in behaving animals in vivo [23-25]. In contrast to BOLD-sensitive MRI sequences, $\mathrm{Mn}^{2+}$-enhanced MRI completely avoids the occurrence of image artifacts from magnetic susceptibility differences within the head (Section 3.4.2). Suitable T1-weighted MRI sequences allow for three-dimensional (3D) acquisitions at high spatial resolution several hours after contrast administration.

Other functional MRI studies focus on the migration of stem cells in animal brain in vivo [26] after labeling with superparamagnetic iron-oxide nanoparticles [27]. Similarly, monoclonal antibodies linked to iron-oxide particles or more conventional paramagnetic contrast agents are used to characterize the distribution of receptors by MRI [28,29]. Even the expression of genes was demonstrated using reporter genes co-expressing a contrast-enhancing compound for MRI [30,31]. This potential has initiated a broad development of MRI-based techniques for in vivo imaging of gene expression, for example, see Refs. [32-34].

\section{Basic elements}

The purpose of this section is to discuss the general outline of a functional MRI study. It covers the basic ideas and mechanisms that allow for the observation of blood flow-mediated responses to a functional challenge by a deoxyhemoglobin-sensitive MRI recording. The section discusses selected aspects of data acquisition, data analysis, statistical treatment, and generation of an activation map. A typical experiment dealing with the functional organization of the visual cortex serves to illustrate the design of a robust activation study in individual human subjects.

\subsection{The principle}

Similar to other noninvasive methods which monitor brain function by recording associated electromagnetic 
fields, that is, electroencephalography (EEG) [35,36] and magnetoencephalography [37], magnetic resonance functional neuroimaging represents an indirect measure of neural activation based on cerebral perfusion. In more detail, the intra- and extracellular events following a change of activity in the circuitry of a reasonably large population of neurons transform into hemodynamic and metabolic changes, which affect the intravascular level of (deoxygenated) hemoglobin in the local microvasculature. In contrast to the diamagnetic oxyhemoglobin complex, deoxyhemoglobin is a paramagnetic molecule. It may therefore be exploited as an endogenous contrast agent for T2*-weighted gradient-echo MRI and used as an indicator of activation-induced changes in cerebral blood flow and metabolism. In physical terms, BOLD MRI signal changes are primarily due to deoxyhemoglobin-related susceptibility effects, which alter the microscopic magnetic field gradients in and around capillaries, venules, and veins. The BOLD contrast is therefore composed of both an intra- and extravascular component. Their relative contributions depend on the static magnetic field strength, the echo time of the imaging sequence, and the nature of the MRI signal, that is, a gradient echo probing dephasing effects or a spin echo probing diffusion effects.

An important point to emphasize is the fact that functional MRI detects changes of brain activity rather than brain activity per se. As a consequence, the approach does not allow for an absolute quantitation of brain activity, at least not when based on deoxyhemoglobin-sensitive MRI sequences or BOLD contrast. Instead, serial MRI recordings during brain activation attempt to identify relative differences between successive functional states of the subject under investigation. In other words, magnetic resonance mapping of brain activation refers to the dynamic acquisition of signal intensity changes which accompany the transition from one state of brain activity to another.

Taken together, the practical concept underlying a functional MRI experiment starts with the design of a paradigm, which switches between at least two different functional states. Although the paradigm design appears simple for studying primary functions such as vision and sensorimotor processing, it presents a problem of increasing complexity when asking for a specific and objective cognitive (or even emotional) effort in higher-order processing. Moreover, the paradigm needs to be translated into a stimulus protocol which takes into account the temporal profile, that is, the time constants, of BOLD MRI signal changes in order to match the major hemodynamic characteristics of the cerebrovascular system and to improve the detectability of small and/or transient responses. The parameters chosen for data acquisition have to be optimized with respect to the desired spatiotemporal resolution and the achievable functional contrast, while the data analysis commonly relies on the assumption of a distinct (temporal) relationship between a functional challenge and an associated MRI signal deflection in a particular brain region or image voxel. Even then, additional precautions are required before exclusively assigning all statistically validated spots in an activation map to cortical processing of the original task.

\subsection{The mechanism}

Brain activation commonly refers to enhanced (or suppressed) neural firing and axonal spiking. In the associated cascade of cellular events the mechanisms supporting synaptic transmission are likely to represent the most relevant energy-consuming processes as they regulate the release and uptake of neurotransmitters. These ATP-dependent processes are the main candidates for stimulating the elevation of blood flow and associated substrate delivery (oxygen and glucose). The mechanism involves a variety of molecular mediators which selectively act on the smooth muscles of arterioles feeding the active population of neurons [38]. Because of this neurovascular and neurometabolic coupling, neural activity changes affect the regional cerebral (arterial) blood flow, the cerebral (venous) blood volume, and the oxidative glucose consumption.

Although it is possible to directly detect activationinduced changes in brain perfusion by monitoring altered blood flow by using arterial spin tagging [14-16], the vast majority of applications exploits the sensitivity of gradientecho MRI sequences to changes in magnetic susceptibility which are due to dynamic differences in blood oxygenation, or more precisely, the intravoxel concentration of paramagnetic deoxyhemoglobin in red blood cells.

In terms of MRI contrast, it is the absolute level of deoxyhemoglobin that determines the susceptibilityinduced signal reduction of the water proton signal in any particular image voxel. Apart from the hematocrit, which refers to the total number of red blood cells, the deoxyhemoglobin concentration is modulated by both hemodynamic and metabolic processes which therefore contribute to the resulting deoxyhemoglobin-based MRI signal strength. A more detailed quantitative analysis of relative amplitudes and time constants not only requires further experimental insights but is also outside the scope of this article. Briefly, however, the current 'consensus model' of BOLD MRI changes in response to a functional challenge assumes a major increase in cerebral blood flow upon activation, that is, for increased neural activity. In fact, the excess delivery of oxyhemoglobin by elevated blood flow emerges as the predominant parameter for MRI as it effectively decreases the absolute deoxyhemoglobin concentration-predominantly in the venous part of the microvasculature. The resulting MRI signal increase is only partly counterbalanced by a slight increase of the deoxyhemoglobin level arising from enhanced oxygen consumption and increased cerebral blood volume.

The aforementioned phenomena have led to a variety of theoretical descriptions. In particular, Buxton and Frank [39] 
addressed the relationship between blood flow and oxygen delivery to brain tissue and developed a Balloon (or Windkessel) model of venous compliance [40] which couples the hemodynamic outflow to blood volume changes and hence the BOLD response [41]. The relevance of elevated oxygen consumption was stressed by Hoge and colleagues $[42,43]$. These authors characterize the BOLD effect as the outcome of strong competition between oxygen consumption and flow adjustment reflected in the net concentration of deoxyhemoglobin. Further, recent descriptions comprise the design of a nonlinear system linking changes in neural activity to BOLD MRI responses [44] and a refined version of this system [45] which extends the inherent Balloon model to a full dynamic capillary model applicable to both transient and steady-state conditions.

\subsection{Deoxyhemoglobin-sensitive MRI: BOLD contrast}

\subsubsection{Technical aspects}

Generic gradient-echo sequences suitable for dynamically monitoring BOLD MRI signal intensities are EPI [9,10,12] and FLASH [11] (Section 5). Because single-shot EPI (or spiral imaging [46]) offers subsecond speed, multi-slice applications with repetition times of $2-3 \mathrm{~s}$ are generally sufficient to cover a volume encompassing the entire brain. On the other hand, the limited echo train length of an EPI sequence, in particular at high magnetic fields with increasingly shorter $\mathrm{T} 2 *$ relaxation times, limits the number of possible gradient echoes, so that the respective images (and activation maps) exhibit only a moderate to poor spatial resolution. This finding presents a severe limitation because the maximum switching rate of the magnetic field gradients used for echo generation is restricted to avoid peripheral nerve stimulation, for example, see Refs. [47-51].

One way of alleviating the problem is the use of segmented $k$-space acquisitions leading to multi-shot EPI [52,53] or multi-shot spiral imaging [54]. The procedure requires multiple radiofrequency excitations separated by a reasonable repetition time but reduces the influence of susceptibility artifacts by shortening the echo train length as well as the effective mean echo time. High spatial resolution may be achieved at the expense of a much prolonged measuring time by combining multiple acquisitions each with only partial (segmented) $k$-space coverage. Foreseeable improvements are to be expected from an adaptation of parallel imaging strategies [55-58] as such techniques allow for a reduction of the penalty in image acquisition time.

Complementary to multi-shot EPI, multi-echo FLASH sequences may be employed for acquisitions at high spatial resolution. These techniques bear the additional advantage of reducing image sensitivities to both susceptibility losses and geometric distortions which in EPI are caused by differential resonance offset effects along the echo train. Of course, similar to EPI (and all other MRI sequences), parallel imaging techniques will improve FLASH MRI as well. For high-resolution studies with BOLD contrast the potential benefits mainly translate into a significantly reduced measuring time.

Beyond the mainstream approaches discussed so far, there are BOLD MRI studies based on spin-echo EPI sequences mainly at high magnetic fields of $4 \mathrm{~T}$ and $7 \mathrm{~T}$, for example, see Ref. [59]. Such techniques have been proposed to enhance the sensitivity to deoxyhemoglobin changes in small vessels relative to contributions from larger veins. The underlying rationale is to emphasize BOLD-induced diffusion changes in $\mathrm{T} 2$ as opposed to the pronounced dephasing effects reflected in $\mathrm{T} 2 *$. The idea is based on Monte Carlo simulations [60,61] of the dynamic behavior of deoxyhemoglobin within a brain image voxel which suggest that the spin-echo BOLD MRI signal at high but not low magnetic fields is predominantly sensitive to the microvasculature of the brain parenchyma. However, recent numerical results for a vascular network model of randomly oriented paramagnetic vessels [62] indicate that for the optimal echo time the extravascular contribution of the BOLD MRI signal increases with field strength for both the gradient-echo and spin-echo signal. Moreover, the simulations demonstrate that the extravascular contributions of capillaries represent only about $10 \%$ of the total BOLD effect for gradient echoes and $20 \%$ for spin echoes, even at 7T [62]. Apart from the dependence of numerical simulations on physiologic parameters such as the distribution of vessel sizes, and despite the fact that functional MRI sequences may have slightly different biases toward larger or smaller blood vessels, a separation of intra- and extravascular effects will neither be total nor may it be experimentally proven. In fact, the spin-echo EPI sequences used so far exhibit a significant $\mathrm{T} 2{ }^{*}$ sensitivity due to the large number of gradient-echo signals which occur at variable echo times relative to the central spin echo. Although the range of gradient-echo times may be shorter in a spin-echo EPI sequence than for a pure gradient-echo EPI sequence, the much shortened $\mathrm{T} 2 *$ relaxation times at high magnetic fields still lead to a substantial gradient-echo effect which tends to dominate the acquired BOLD phenomenon.

On the other hand, the results of high-resolution BOLD MRI studies at 1.5-2.0 T indicate a tight spatial control of activation-induced changes in blood flow, so that suitable approaches provide access to functional columns (Section 3.2.1). It may therefore be concluded that early concerns derived from near-infrared optical imaging [63] about a potential spread or 'vascular blurring' of BOLD MRI activations beyond the region of true neural activation may have been exaggerated and probably are of limited practical relevance-even for submillimeter MRI voxel dimensions. In addition, a certain degree of spatial overlap may be effectively eliminated by differential paradigms which ask for quantitative rather than qualitative differences between the cortical representations of functional states (Section 3.6.2). 


\subsubsection{Physiological aspects}

Fig. 1 shows mean BOLD MRI responses averaged across subjects for both a single $10 \mathrm{~s}$ visual stimulation period contrasted by a dark screen and a corresponding repetitive protocol with $20 \mathrm{~s}$ interstimulus intervals [64]. The response curves directly reflect the various mechanisms and respective time courses affecting the deoxyhemoglobinbased BOLD MRI signal. Immediately after stimulus onset, the response to a $10 \mathrm{~s}$ stimulus with a $90 \mathrm{~s}$ recovery period reveals a latency period of about $1-2 \mathrm{~s}$ before the pronounced elevation of blood flow reduces the deoxyhemoglobin level and thereby increases the BOLD MRI signal. For the chosen spatial resolution and a strong visual stimulus such as a black and white reversing circular checkerboard, which activates almost all populations of specialized neurons in the visual cortex, the observable
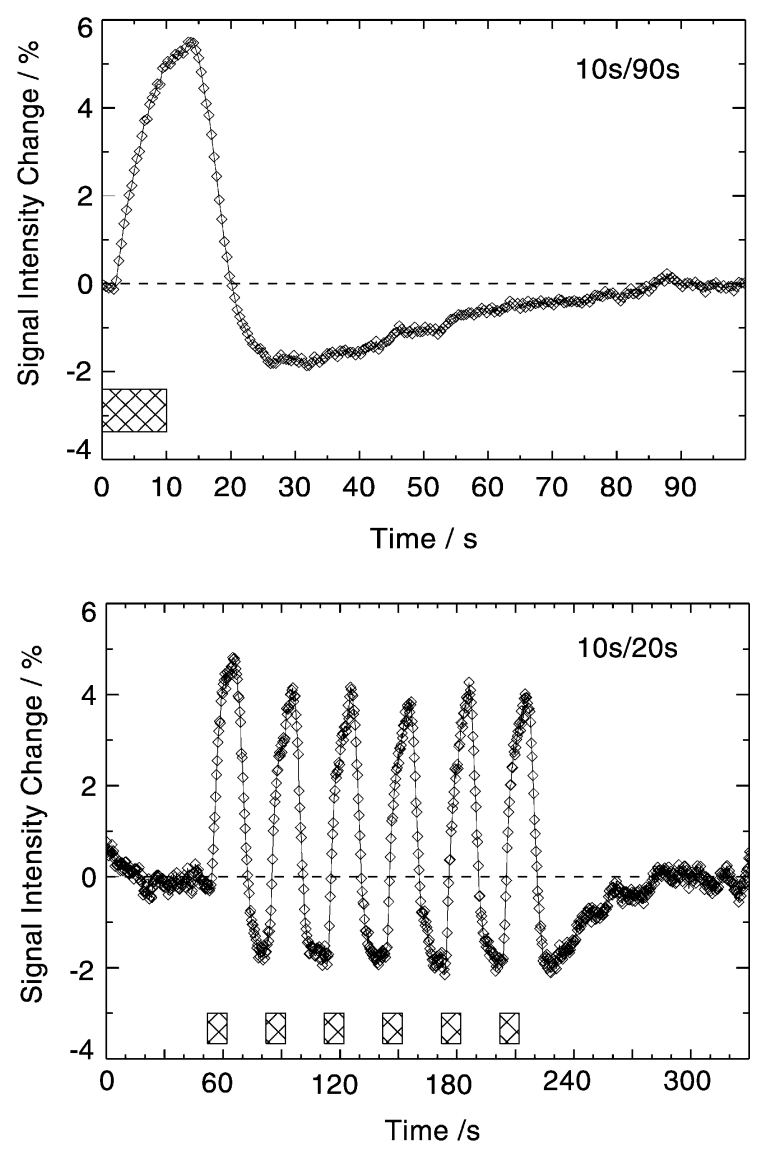

Fig. 1. Functional MRI responses elicited by visual stimulation. The data represent mean BOLD MRI signal intensity time courses averaged across subjects $(n=7)$. The paradigm contrasted a $10 \mathrm{~s}$ presentation of a reversing checkerboard with (top) a 90 s presentation of a dark screen and (bottom) a $20 \mathrm{~s}$ dark screen in a repetitive protocol (single-shot EPI, TR/TE $=400 / 54$ $\mathrm{ms}$, flip angle $30^{\circ}$, spatial resolution $3 \times 3 \times 4 \mathrm{~mm}^{3}$ ). The response to a single $10 \mathrm{~s}$ stimulus is characterized by an initial brief latency, a pronounced BOLD MRI signal increase, a post-stimulation undershoot, and a $60 \mathrm{~s}$ recovery period. These latter events overlap with subsequent positive BOLD MRI responses during repetitive stimulation. The resulting 'drift' of the MRI baseline signal recovers to the true pre-stimulation baseline after the end of repetitive stimulation. Adapted from Ref. [64] with permission. maximum signal increase is on the order of $6 \%$. Longer stimuli yield a flattening response curve which may even decrease in amplitude for sustained activations with durations longer than about $30 \mathrm{~s}$. Part of this signal reduction may be caused by the slightly delayed development of a signal undershoot which becomes unmasked in the post-stimulation period. During this phase the rapid normalization of blood flow eliminates the strong supply of oxyhemoglobin so that the slower and still persistent increase of the blood volume above prestimulation level leads to a net increase in deoxyhemoglobin. For the experiment shown in Fig. 1 this effect causes a signal decrease of up to $2 \%$ below the pre-stimulation baseline. Noteworthy, the elevated blood volume requires a period of about $60 \mathrm{~s}$ to completely recover. It is therefore of no surprise that repetitive stimulation protocols reveal response curves that represent the overlap of a positive BOLD MRI response to the actual stimulus with undershoot effects from preceding stimuli.

The lower part of Fig. 1 shows that the rapid succession of blood flow-mediated signal increases and blood volumerelated signal decreases generates a steady-state BOLD MRI response with an apparent baseline below the pre-stimulation level. As expected, the underlying undershoot phenomenon only recovers after the very end of the protocol within a period of about $60 \mathrm{~s}$. Interestingly, most researchers miss these physiologically determined response characteristics as they tend to restrict their stimulus protocols to relatively rapid transitions of brain activity without defining a baseline condition. The full response curve also demonstrates that the BOLD contrast benefits from both the positive signal increase and the negative undershoot, so that a proper timing of the protocol may exploit these phenomena to optimize the functional contrast between conditions.

\subsection{Correlation analyses and beyond}

The temporal characteristics of the BOLD MRI response curves lend themselves to a voxel-by-voxel temporal correlation analysis [65]. Such strategies correlate MRI signal intensity time courses with a reference function that mimicks the paradigm or stimulation protocol. Often these reference functions represent simple boxcar ('On-Off') waveforms which do not attempt to simulate the actual hemodynamic characteristics apart from a shift by 2-6 s with respect to the actual time course, that is, by one or two images depending on the temporal sampling as defined by the repetition time. It is of course possible - and in eventrelated designs certainly advantageous-to perform a convolution of the rectangular waveform with a suitable hemodynamic response function (Section 3.3.1). The procedure allows one to compensate for the initial hemodynamic latency and the subsequent rise (and fall) of the BOLD MRI signal.

Following the calculation of a parametric map based on correlation coefficients, the definition of objective 
and effective thresholds is a crucial step in the analysis of paradigm-related activations. Although fixed correlation coefficients seem to be popular thresholds, they do not represent objective levels of confidence. For example, their values strongly depend on the length of the acquired fMRI time series, that is, the number of images or data points contributing to the correlation analysis [66]. In other words, when the duration of the fMRI experiment is doubled while all other parameters remain the same, the resulting correlation coefficient of a selected, truly activated pixel decreases. Or conversely, for a shorter fMRI experiment with less images such correlation coefficients increase. Similar statistical problems arise for comparisons involving images with a different number of pixels. Accordingly,
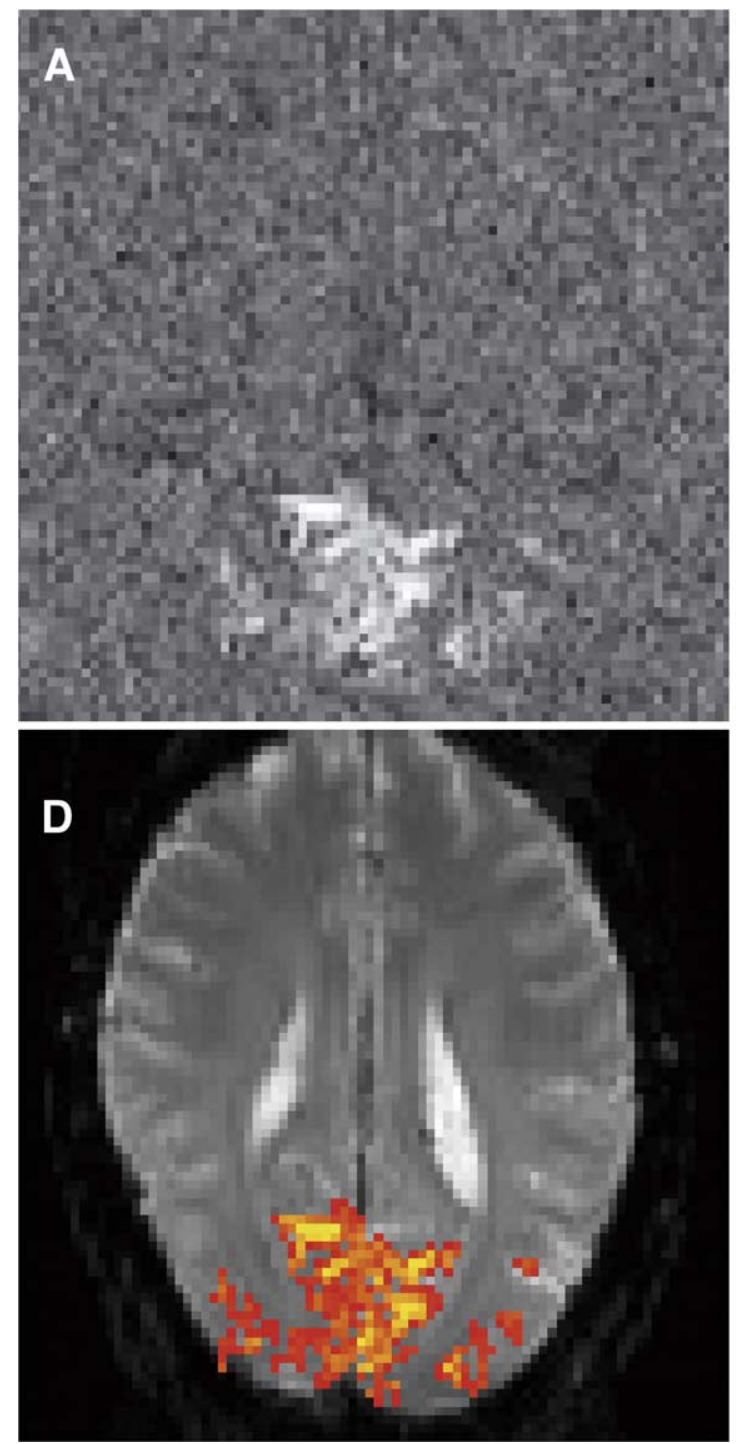

the use of identical correlation coefficient thresholds in fMRI experiments comprising different numbers of images or images with a different pixel resolution is by no means statistically justified. Instead, the procedure should involve the application of a probabilistic threshold indicating to which degree a high correlation coefficient occurs by chance or represents a significant effect. In line with earlier suggestions and a wide acceptance in test statistics, it is therefore strongly recommended to use $p$ values which preferentially are defined with respect to the physiological noise distribution of the individual maps.

Fig. 2 outlines the strategy taken in this laboratory. The gray-scale image in Fig. 2A represents a native map of correlation coefficients, that is, values of the correlation
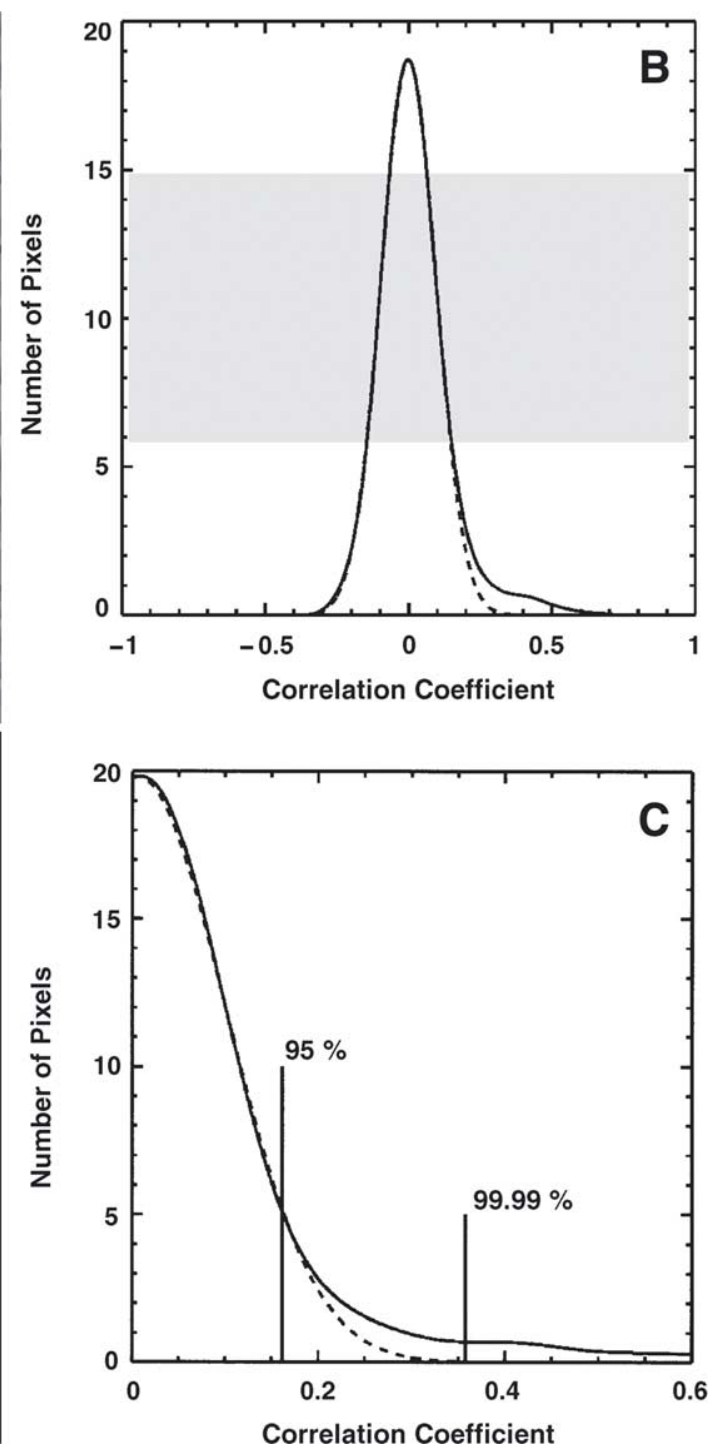

Fig. 2. Correlation analysis and statistical evaluation of functional MRI responses to human brain activation. (A) Correlation coefficient map (no filtering) obtained for visual stimulation (checkerboard vs gray screen, single-shot EPI, TR/TE $=2000 / 54 \mathrm{~ms}, 70^{\circ}, 2 \times 2 \times 4 \mathrm{~mm}^{3}$ ). The gray-scale intensities represent correlation coefficients ranging from -1 (black) through 0 (gray) to +1 (white). (B) Histogram of the correlation coefficient map (solid curve) used to estimate the underlying noise distribution by a symmetric Gaussian function (broken curve). The shaded area indicates the fitted part of the histogram (30-80\% of the maximum height). (C) Renormalization of the correlation coefficient distribution into percentile ranks of the noise distribution allows one to identify centers of activation above an upper threshold of $99.99 \%$. Neighboring voxels are iteratively added as long as their correlation coefficient exceeds a lower threshold of $95.00 \%$. (D) Thresholded and color-coded activation map superimposed onto an original echo-planar image. 
coefficient function at zero lag time, which was obtained without any spatial or temporal filtering. In order to derive a statistically evaluated activation map by an objective and automated analysis, the histogram of all correlation coefficients is analyzed with respect to deviations from a symmetric noise distribution. Numerous trials of correlation analyses of serial MRI data acquired in the absence of a stimulus have shown that respective correlation coefficient histograms may be represented by a symmetric (Gaussian) function [67]. In addition, such experiments revealed a considerable variety of the histogram broadness even for intrasubject comparisons. While this observation precludes the use of absolute correlation coefficients as meaningful thresholds, it also points to the physiological character of the BOLD MRI signal fluctuations in the 'resting' brain. Most likely, the physiological noise reflects both differences in systemic circulation such as blood pressure and uncontrolled cortical activity not related to the actual task.

As shown in Fig. 2B, the noise distribution (broken line) embedded within the histogram of a mapping experiment is estimated by fitting a Gaussian function to a central region extending from 30 to $80 \%$ of the maximum height (shaded area). While the upper central part of the histogram eventually deviates from a smooth behavior, for example, for low-resolution scans with a smaller total number of image voxels, the lower part contains positive deviations from the noise distribution that represent activated voxels. Fig. 2C demonstrates that voxels become automatically accepted as activated above an upper threshold of $99.99 \%$ of the integrated noise distribution. The underlying concept corresponds to a renormalization of the correlation coefficients into percentile ranks of the noise distribution. This conservative procedure identifies activated centers but does not cover the full extent of activated voxels as easily recognized by inspecting the difference of the actual histogram and the estimated noise distribution. Thus, to further delineate the spatial extent of activated centers, directly neighboring voxels are iteratively added as long as their correlation coefficients exceed a lower threshold chosen to be the $95 \%$ percentile rank. The resulting color-coded activation map in Fig. 2D compares favorably with the original correlation coefficient map shown above.

Altogether the described automated and user-independent procedure [66] has been successfully applied to a large number of studies over several years. It is transparent, largely driven by the actual data, and provides a robust and objective way of thresholding statistically evaluated parametric maps on an individual basis. Moreover, it improves the sensitivity without essential loss of specificity. This is accomplished by using a combination of two distinct $p$ values for identifying activation foci and defining the full spatial extent through neighborhood relations, respectively.

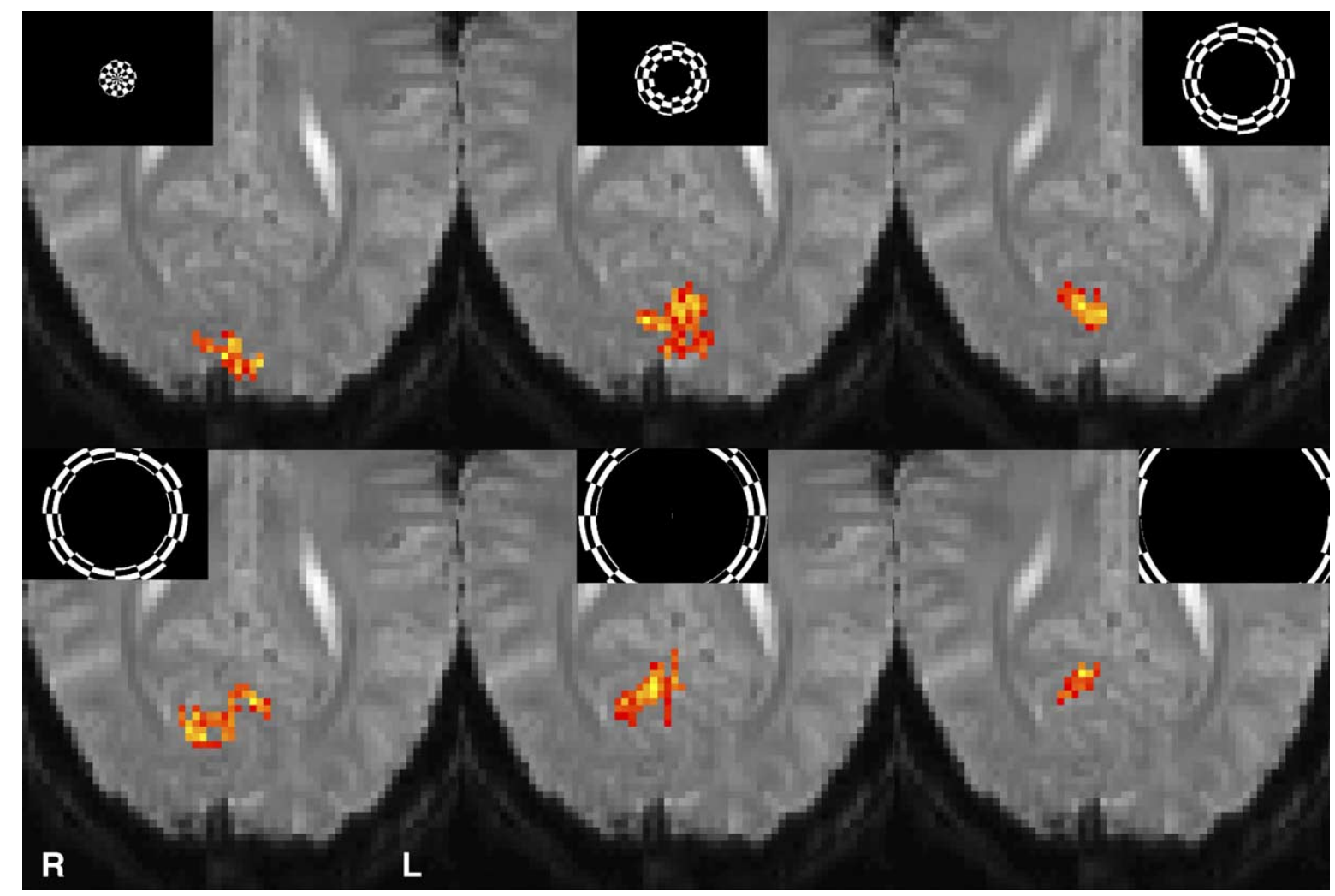

Fig. 3. Functional MRI of the retinotopic organization of the human visual cortex: eccentricity. The paradigm consists of six successive $10 \mathrm{~s}$ presentations of rings with increasing diameter (inserts) expanding over a $30^{\circ} \times 40^{\circ}$ visual field (other parameters as in Fig. 2, L: left and R: right hemisphere). Within the same section, the color-coded activations move from the occipital pole (representation of the fovea: central viewing) into more anterior locations (peripheral viewing). 
A general advantage emerges from the fact, that correlation analyses are also capable of detecting negatively correlated events. Such 'deactivations' refer to relative increases of the deoxyhemoglobin level which may arise because of a number of reasons, for example, by switching from a state of high to low functional activity in a 'reversed' paradigm [68]. More commonly, the level of activity in one particular brain system (area) becomes modulated (decreased) because of pronounced processing in another brain system (area). A typical example for the latter case is the occurrence of reduced activity in the precuneus and posterior cingulum during attention to a hand motor task [69]. Competitive cognitive processing and attentional modulation of perception are of major scientific interest and the subject of numerous functional brain mapping studies. Finally, a qualitatively different category of MRI signal decreases may arise from pathological alterations of blood flow (Section 3.5.2)

\subsection{A typical experiment: retinotopic organization of the visual cortex}

Rather than contrasting only two functional states, physiologically optimized MRI experiments offer more efficient ways of encoding multiple functional states for mapping. The example shown in Fig. 3 relates to the retinotopic organization of the visual cortex, that is, the encoding of ordered and spatially segregated representations of different parts of the visual field on the surface of the occipital lobe. The paradigm consists of $10 \mathrm{~s}$ presentations of six rings with increasing diameter (stimuli as inserts) expanding over the subject's visual field from its center to the periphery. Data acquisition involved a single-shot EPI sequence at $2 \times 2 \mathrm{~mm}^{2}$ resolution and $4 \mathrm{~mm}$ section thickness and lasted for about 4 min using four repetitions of the entire paradigm. During data analysis each ring stimulus was contrasted with all other stimuli, which resulted in differential representations for the various parts of the visual field with increasing eccentricity. Thus, the same data set was analyzed multiple times with different boxcar reference functions each selecting a particular stimulus. Within the same brain section the respective cortical activations move from the occipital pole representing foveal (central) viewing into more anterior positions encoding for peripheral viewing.

A complementary study shown in Fig. 4 maps the representations of visual field segments in the visual cortex. The paradigm consists of $60^{\circ}$ wedges (stimuli as inserts) rotating through the subject's visual field. When based on differential analyses with all other stimuli, segments in

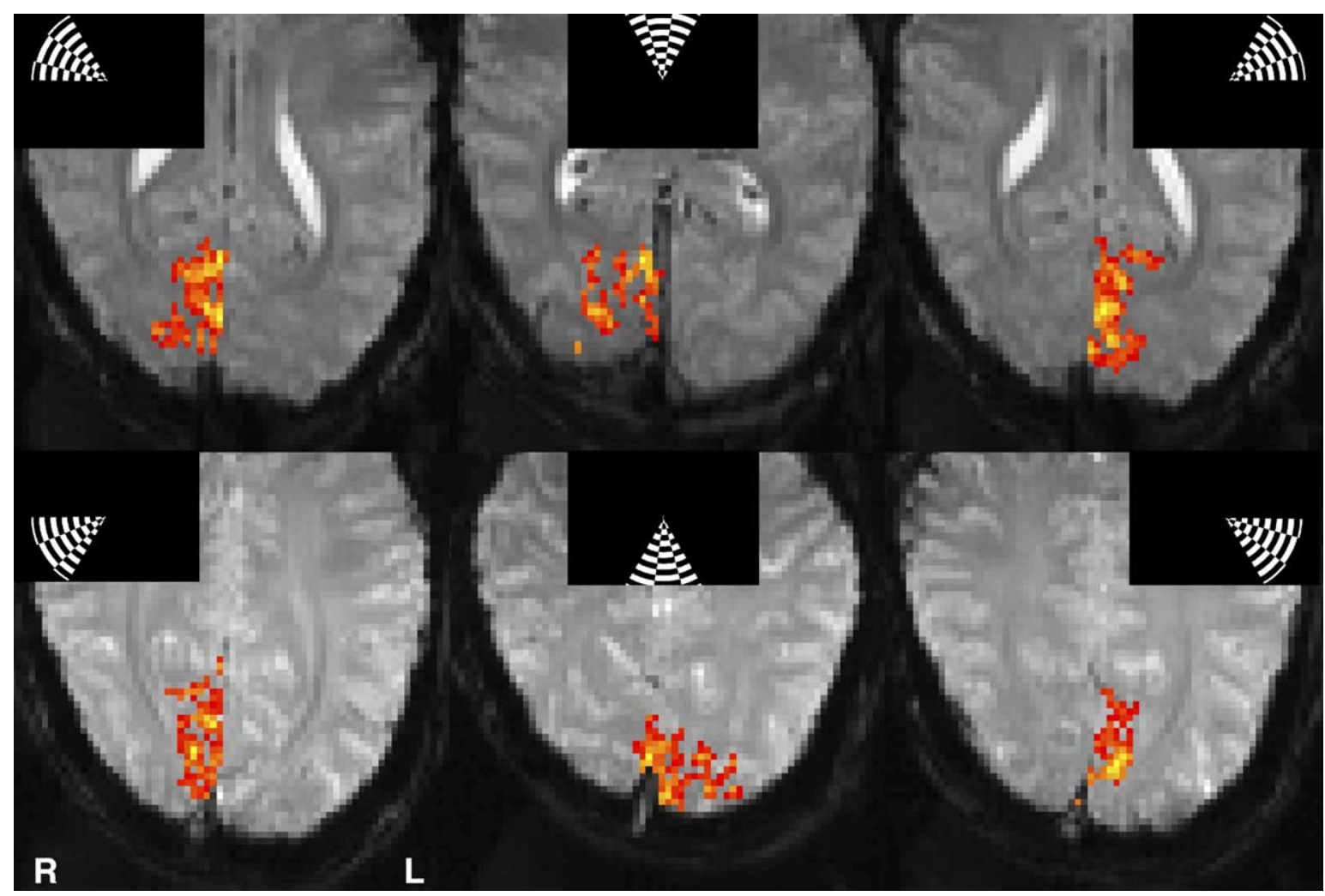

Fig. 4. Functional MRI of the retinotopic organization of the human visual cortex: visual fields. The paradigm consists of six successive $10 \mathrm{~s}$ presentations of $60^{\circ}$ wedges (inserts) rotating through a $30^{\circ} \times 40^{\circ}$ visual field (other parameters as in Fig. 2, L: left and R: right hemisphere). Whereas stimulation of the upper visual field (top row) elicits activations in the lower part of the visual cortex, lower visual fields are represented in the upper parts of the visual cortex. The effect is demonstrated by selecting respective sections with a maximum number of activated pixels. In addition, stimulation of the right (right column) and left visual field (left column) causes activations in the respective contralateral parts of the visual cortex. 
the upper visual field in the top row of Fig. 4 elicit activations in the lower part of the visual cortex as evidenced by a maximum number of activated voxels in the sections shown. In contrast, activations of the three lower visual field segments in the bottom row of Fig. 4 are represented in the upper parts of the visual cortex. In addition, in the right and left part of Fig. 4, stimulation of the two segments in either the subject's right or left visual field cause activations in the respective contralateral part of the visual cortex.

\section{Advances and limitations}

Apart from addressing the neural basis of the BOLD MRI signal, this section discusses the chances offered and the challenges posed by practical applications of MRI to mapping brain function. In particular, this applies to the influence of low and high spatial resolution on both data acquisition and data analysis, the achievable temporal resolution and, more importantly, the ability to monitor brief cortical events. For the clinical setting, additional complications will be addressed that may arise from pharmacological and pathological modulations of the hemodynamic responsiveness. Finally, the paradigm design itself needs to be investigated as often even minor changes in the stimulus presentation may qualitatively alter the results by implicitly changing the original scientific question.

\subsection{On the neural basis of BOLD MRI responses}

While a large number of studies have addressed the physiological basis of the BOLD MRI contrast and its temporal evolution under various experimental conditions, under which circumstances and to which degree the hemodynamically mediated responses reflect the underlying changes in focal neural activity are less well understood-for recent reviews see Refs. [70,71]. A specific point refers to the spatial accuracy of BOLD MRI activations as deoxyhemoglobin concentrations may be affected in a larger part of the microvasculature and therefore blur the representation of the truly activated neural population. Moreover, it remains to be established whether the duration of a neural event exhibits a quantitative (or even linear) correspondence with the duration and/or strength of the BOLD MRI signal and whether the temporal relationship between a neural event and its hemodynamic counterpart maintains (small) relative differences. Finally, and most importantly, it needs to be clarified which elements of the processes simplified as neural activation, for example, synaptic transmission or membrane depolarization, are associated with a metabolic and hemodynamic demand and therefore possess the capacity to provoke a change in the deoxyhemoglobin concentration.

The latter problem has recently been tackled using simultaneous electrophysiological recordings and BOLD
MRI acquisitions of visual processing in anesthetized monkeys [72]. Extending studies of activity-dependent increases of cerebral blood flow in conjunction with extracellular recordings of single unit activity and field potentials in rat cerebellar cortex [73], the primate experiments demonstrated that the hemodynamic response correlates better with the local field potential, which is linked to synaptic activity, rather than with the action potential as given by the spiking of individual neurons or groups of neurons. Because local field potentials and multi-unit activity distinguish between different aspects of neural information processing, the BOLD MRI responses are likely to reflect the input and intracortical processing of a given brain area rather than its spiking output-despite the fact that in most cases these events are coupled [74].

This view is supported by studies in humans combining BOLD MRI recordings with transcranial electromagnetic fields known to stimulate brain tissue [75,76]. When a transcranial magnetic stimulation (TMS) coil is positioned close to the skull, the generated time-varying magnetic fields induce electric fields in the cortical tissue which may lead to neuroaxonal depolarization [77]. It turns out that the combination of two circular coils into a figure-of-eight arrangement allows one to focus the effects and elicit localized 'cortical activity'. A typical example is evoking unilateral finger movements by TMS of the contralateral primary motor cortex (M1).

Fig. 5 shows BOLD MRI activation maps for four different experimental conditions designed to answer the question of whether or not TMS itself induces MRIdetectable responses [78]. When performing a sequential opposition of all fingers to the thumb (right hand) in conjunction with weak subthreshold TMS of the left motor cortex (SFO 25\%), TMS produced no other observable effects than acoustically perceptible clicks which served to request the actual finger movements. TMS of the left M1 above the motor threshold (M1 110\%) caused finger movements of the right hand as well as clicks, whereas subthreshold TMS of the left M1 (M1 90\%) as well as suprathreshold TMS of the left lateral premotor cortex (LPC 110\%) did not result in finger movements. The data of Fig. 5 (top row) therefore demonstrate that BOLD MRI responses in the primary motor cortex are only observed if subjects perform a real finger movement. In more general terms, any integrative processing of cortical input, which alters the synaptic activity, should generate a related hemodynamic response. This particularly holds true for primary sensory systems and here applies to voluntary motor activity and finger movements induced by supratheshold TMS of M1, while similar responses were detected after peripheral electric stimulation of the median nerve [79]. Conversely, subthreshold TMS or suprathreshold TMS atop the LPC does not automatically lead to activations in the stimulated region despite the fact that the procedure certainly leads to neuroaxonal depolarization. 


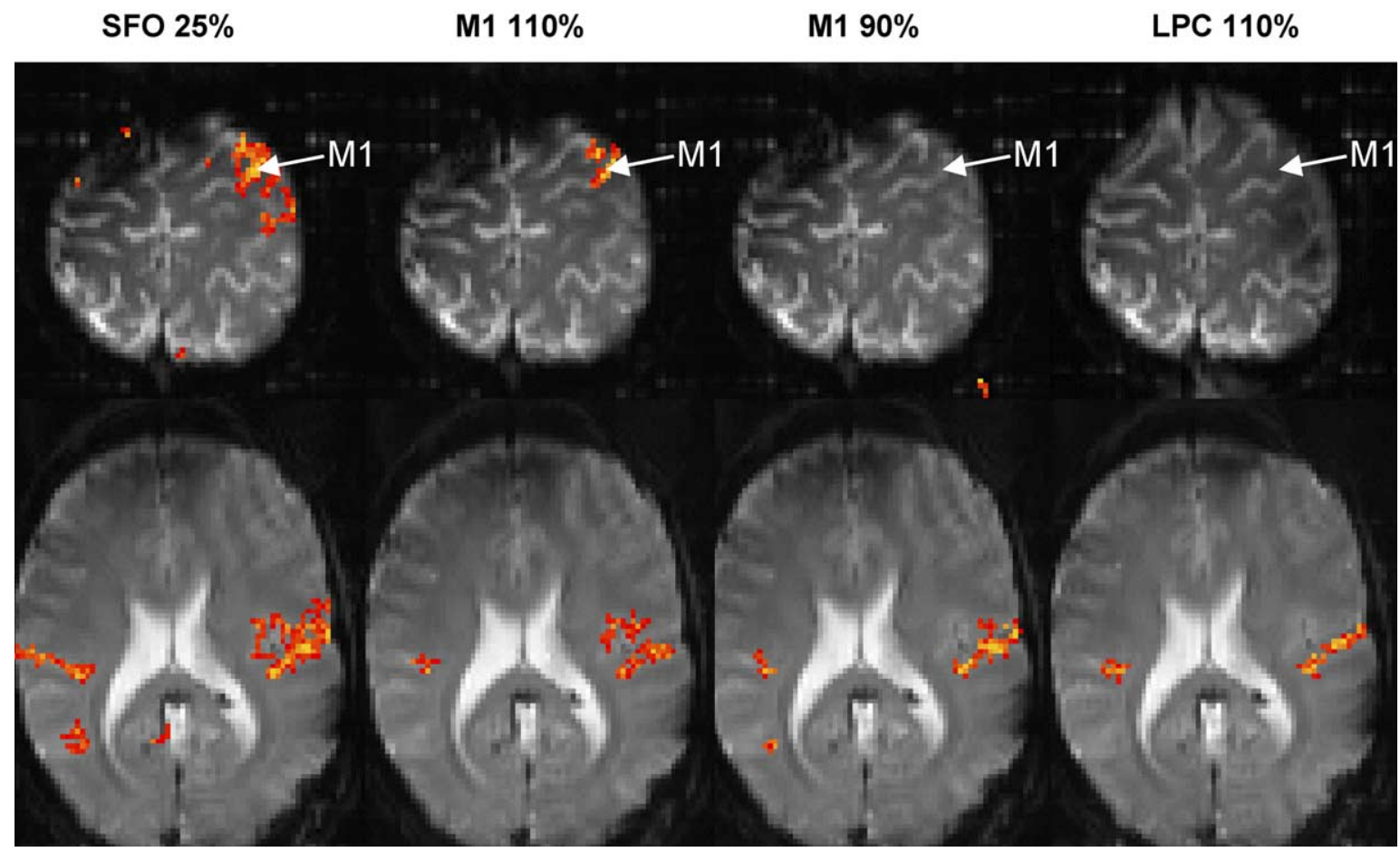

Fig. 5. Functional MRI of voluntary hand movements and transcranial magnetic stimulation (TMS) of the primary sensorimotor cortex hand area. (Top) BOLD MRI activations in the left primary motor cortex (M1) are only observed for actual finger movements either performed voluntarily (SFO $25 \%=$ sequential finger-to-thumb opposition with TMS intensities of only 25\% of the subject's motor threshold) or evoked by suprathreshold TMS (M1 $110 \%=110 \%$ motor threshold). No such activations are elicited by subthreshold TMS of the left motor cortex (M1 90\% $=90 \%$ motor threshold) or suprathreshold TMS of the left lateral premotor cortex (LPC $110 \%=110 \%$ motor threshold with the TMS coil positioned atop the LPC). (Bottom) In all conditions, the clicks of the TMS discharges lead to bilateral activations in the auditory cortex (single-shot EPI, TR/TE $=2000 / 54 \mathrm{~ms}, 70^{\circ}, 2 \times 2 \times 6 \mathrm{~mm}^{3}$ ). Adapted from Ref. [78] with permission.

Extending the aforementioned TMS work and catalyzed by technical advances of combined repetitive TMS (rTMS) and functional EPI [80], short trains (10 s) of high-frequency rTMS $(4 \mathrm{~Hz})$ are indeed able to modulate interconnected frontal motor areas remote from the site of M1 stimulation and even at subthreshold intensity, that is, in the absence of overt motor responses [81]. Using a novel strategy for synchronizing high-frequency rTMS with multi-slice EPI, Fig. 6 demonstrates that subthreshold rTMS $(90 \%$ active motor threshold $=$ AMT $)$ evoked BOLD MRI responses in premotor-primary motor cortical circuits comprising signal increases in the supplementary motor area (SMA) and, to a lesser extent, bilateral LPC as well as signal decreases in the contralateral sensorimotor cortex (M1/S1). These results may be explained either by modulation of post-synaptic activity via direct activation of projecting fibers or indirect secondary modulation of interconnected brain regions. In addition, BOLD MRI activations elicited by suprathreshold rTMS predominantly reflect synaptic processing of both afferent somatosensory and cortico-cortical information. This concept is in excellent agreement with the aforementioned neurophysiological model of the BOLD MRI signal [72,74]. It may also help to further clarify recent observations of reduced BOLD MRI activations in SMA but not M1 after transcranial direct current stimulation [82].

\subsection{Spatial resolution}

The choice of spatial resolution is an important issue in planning a functional MRI study of human brain activation. During data acquisition the voxel size determines the signal-to-noise ratio (SNR), the functional contrast-to-noise ratio (CNR), and the degree of susceptibility-induced signal losses and geometric distortions. Thus, apart from merely blurring or resolving activations within the brain system under investigation, the experimental acquisition parameters manipulate the outcome of the mapping study by additional means. For example, low spatial resolution may completely hamper the detectability of brain regions close to air-tissue interfaces or compromise the CNR by partial occupation with nonactivated tissue (for example, white matter) to such a degree that it affects the correlation analysis. Moreover, during post-processing, the use of spatial (or temporal) filtering as well as the transformation of activation maps into a standardized brain atlas for intersubject averaging may further compromise the results.

In most applications so far, the voxel resolution corresponds to linear dimensions of $2-4 \mathrm{~mm}$ and a section thickness of 3-6 mm. Such conditions allow for mapping of the gross functional organization of most brain systems and their respective networks as, for example, shown in Figs. 3 and 4 for specific aspects of visual processing. Fig. 7 
$110 \%$ RMT
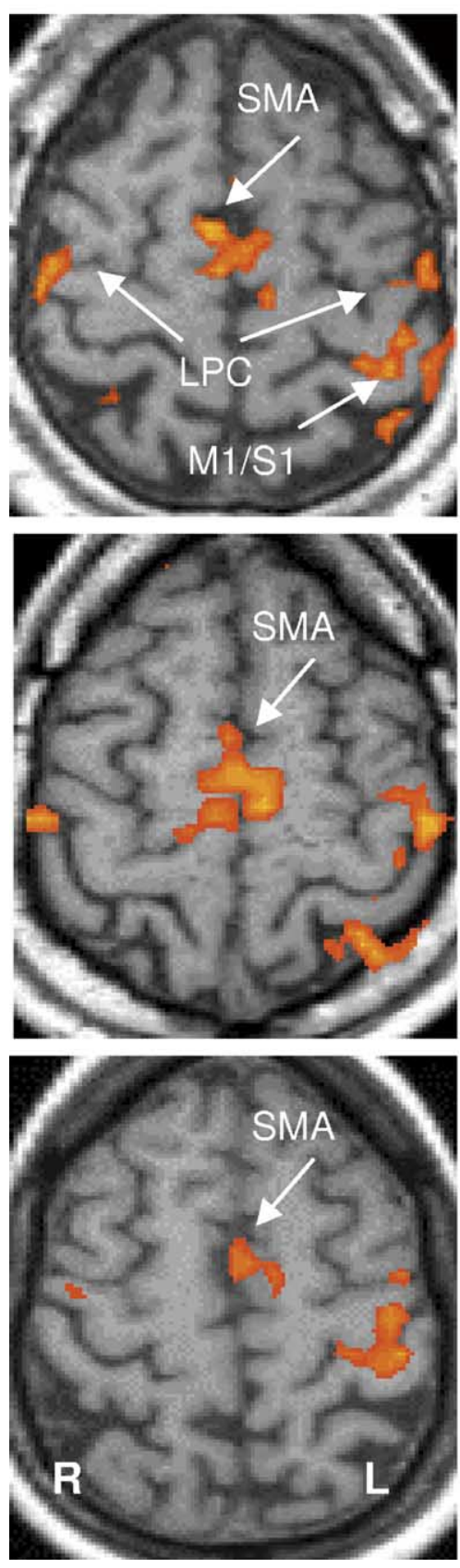

$90 \%$ AMT
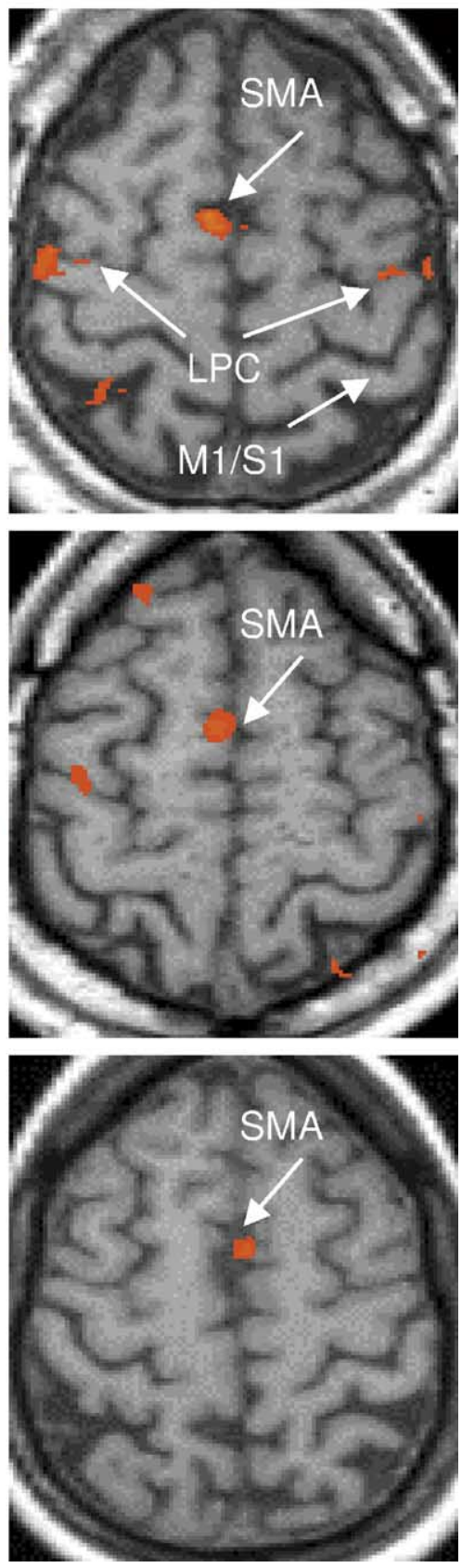

Fig. 6. Functional MRI of repetitive transcranial magnetic stimulation (rTMS) at (left) suprathreshold and (right) subthreshold intensity. Repetitive TMS of the left primary sensorimotor cortex hand area (M1/S1) was conducted in a block design (8 cycles) alternating rTMS epochs (4 Hz, $10 \mathrm{~s})$ with periods of motor rest (20 s) at (left) suprathreshold intensity (110\% resting motor threshold) and (right) subthreshold intensity (90\% active motor threshold). While suprathreshold rTMS evoked activations in M1/S1, supplementary motor area (SMA), and lateral premotor cortex (LPC), subthreshold rTMS elicited responses in SMA but not in the stimulated left M1/S1 (L: left and R: right hemisphere). With TMS pulses applied directly after termination of the EPI acquisition period, the procedure yielded sufficiently long waiting periods before subsequent acquisitions to avoid rTMS-induced image artifacts. Adapted from Ref. [81] with permission.

provides a more detailed comparison of visual activation maps obtained as a function of spatial resolution. It turns out that a $3 \times 3 \mathrm{~mm}^{2}$ in-plane resolution leads to considerable blurring relative to cerebral structures detectable in high-resolution anatomical scans. This finding also applies to the treatment of MRI signals from larger vessels and even includes the sagittal sinus. Much better results are obtained for $2 \times 2 \mathrm{~mm}^{2}$ resolution. While the necessary number of phase-encoding steps can still be matched with the available echo train length of a single-shot EPI acquisition, the resulting image quality is compatible with multi-slice recordings and EPI repetition 

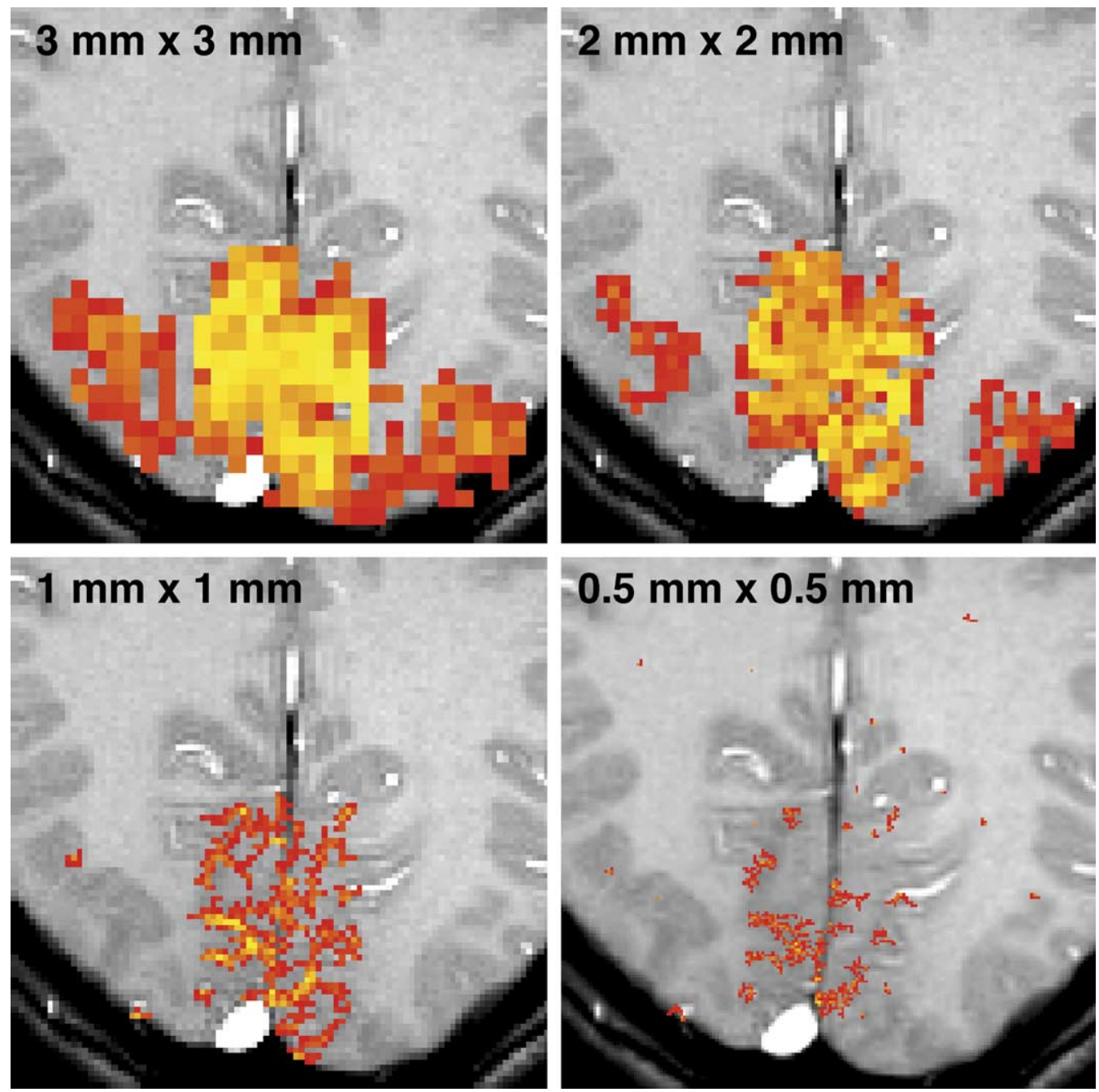

Fig. 7. BOLD MRI activations as a function of spatial resolution. (Top) Visual activation (checkerboard vs gray screen) obtained for EPI at $3 \mathrm{~mm}$ and $2 \mathrm{~mm}$ inplane resolution as well as for (bottom) multi-echo FLASH MRI at 1 and $0.5 \mathrm{~mm}$ resolution (4 $\mathrm{mm}$ sections). Decreasing the voxel size reduces partial volume effects with nonactivated tissue and therefore restricts activations to cortical gray matter at the expense of reduced SNR.

times of 2-3 s. It therefore serves as a 'workhorse' sequence in this laboratory for whole-brain mapping.

An unambiguous restriction of activations to cortical gray matter is only achieved for a spatial resolution of $1 \times 1 \mathrm{~mm}^{2}$. At the expense of reduced volume coverage, the multi-echo FLASH approach yields high-quality activation maps while maintaining adequate sensitivity. In the extreme case of $0.5 \times 0.5 \mathrm{~mm}^{2}$, however, the acquisition has to be reduced to a single section with additional compromises in SNR. Nevertheless, even a reduction in voxel size from 36 to $1 \mathrm{~mm}^{3}$ still yields extended areas of activation. The reason for this observation is an enhanced CNR after removal of partial volume effects with nonactivated tissue [83]. The effect is clearly demonstrated in Fig. 8 comparing the BOLD MRI response strengths for the activated voxels shown in Fig. 7. When defined as the mean peak-to-peak difference of successive deflections of the BOLD MRI signal intensity time course, the functional contrast increases from about $6 \%$ at $36 \mathrm{~mm}^{3}$ resolution to almost $15 \%$ at $1 \mathrm{~mm}^{3}$ resolution which partially compensates for the reduced SNR.

\subsubsection{Functional columns}

So far, the exceptional potential of BOLD MRI for mapping human brain function at very high spatial resolution has seldom been exploited. Pertinent examples mainly represent attempts to resolve the columnar organization of the primary visual cortex [84-87]. The largest structures of such functional units refer to the separate processing of incoming information from either the left or right eye. In human striate cortex these elements form neighboring columns of cortical gray matter with a diameter of $0.75-1.0 \mathrm{~mm}$. As shown in Fig. 9 monocular stimulation indeed results in pronounced BOLD MRI signal intensity changes which reflect neural activity specific for a single eye [85]. In agreement with the findings reported in Figs. 7 and 8, the mean BOLD contrast of about $11 \%$ favorably contrasts with the $6 \%$ obtained at lower resolution 

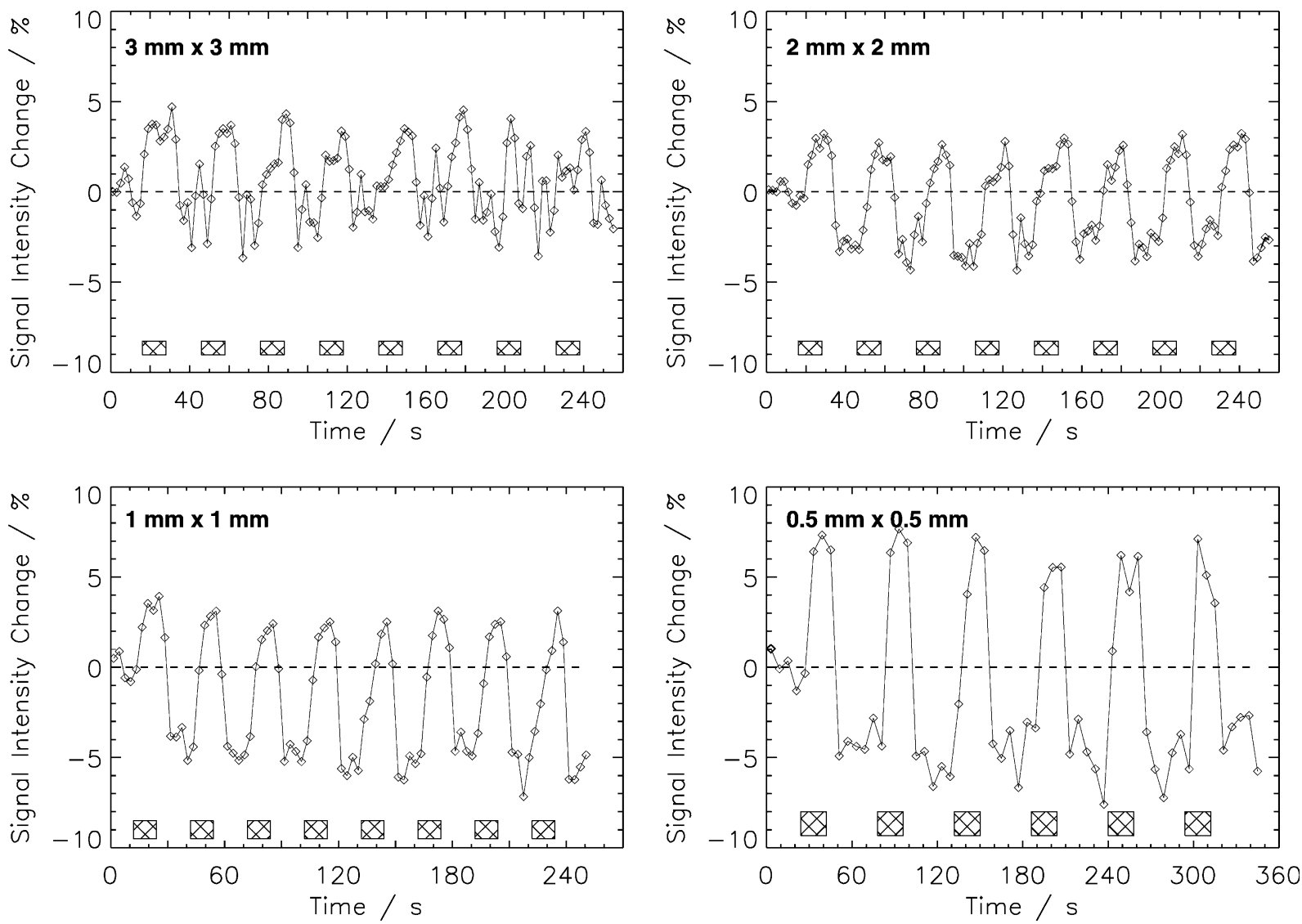

Fig. 8. BOLD MRI contrast as a function of spatial resolution. The reduced SNR expected for acquisitions at very high spatial resolution is partially compensated for by an increase of the functional contrast defined as the peak-to-peak difference of successive deflections of the BOLD MRI signal intensity time course (same data as in Fig. 7). The functional contrast increases from about $6 \%$ at $3 \times 3 \times 4=36 \mathrm{~mm}^{3}$ resolution to almost $15 \%$ at $0.5 \times 0.5 \times 4=1 \mathrm{~mm}^{3}$ resolution by reducing partial volume effects with nonactivated neighboring tissue.

but otherwise similar conditions. The approach yields spatially distributed activations for individual eye representations that closely resemble the neuroanatomical observations of 'frequent bifurcations, blind endings, and islands' found in combined serial sections of specimens from patients blind in one eye [88]. An overlay of the two monocular activation maps proves the complementary nature of right and left eye patterns by the absence of a substantial number of overlapping voxels. The data therefore provide evidence for a direct identification of spatially separated functional units in intact human striate cortex that predominantly code for an individual eye.

\subsubsection{Filtering and multi-subject averaging}

The critical influence of post-processing onto the spatial resolution and potentially also on the scientific outcome of a mapping study is demonstrated in Fig. 10 for the case of visual episodic memory encoding analyzed in different ways. The original data acquisition and analysis at high spatial resolution was performed to reduce susceptibility artifacts and to clarify the involvement of the hippocampus proper [89]. As shown in the sagittal high-resolution map for a single subject, the episodic memory task unambiguously confines activations to the parahippocampal gyrus with no involvement of the hippocampus proper. This finding in all individual subjects was even confirmed after spatial filtering [90]. The procedure employed a statistical parametric mapping (SPM99) software package [91] which generates low-resolution activation maps from realigned, coregistered, and spatially smoothed data with a Gaussian kernel of $8 \mathrm{~mm}$ full width at half maximum height. Nevertheless, filtering merged activations in anatomically and functionally separated brain regions such as the parahippocampal gyrus and the ventral stream of the visual system. Moreover, small foci of activated voxels which originated from veins became blurred and hence indistinguishable from parenchymal responses.

Finally, a multi-subject analysis of activated regions normalized the functional images to an approximate Talairach brain atlas. As shown in the right part of Fig. 10, subsequent intersubject averaging resulted in activation of the left hippocampus proper. This finding could not be confirmed by any of the activation maps obtained at high or low resolution in individual subjects. A possible way to improve the validity of such functional MRI studies may therefore be to acquire the data at adequate resolution to minimize technical artifacts and to additionally analyze the data of individual subjects with and without spatial filtering 

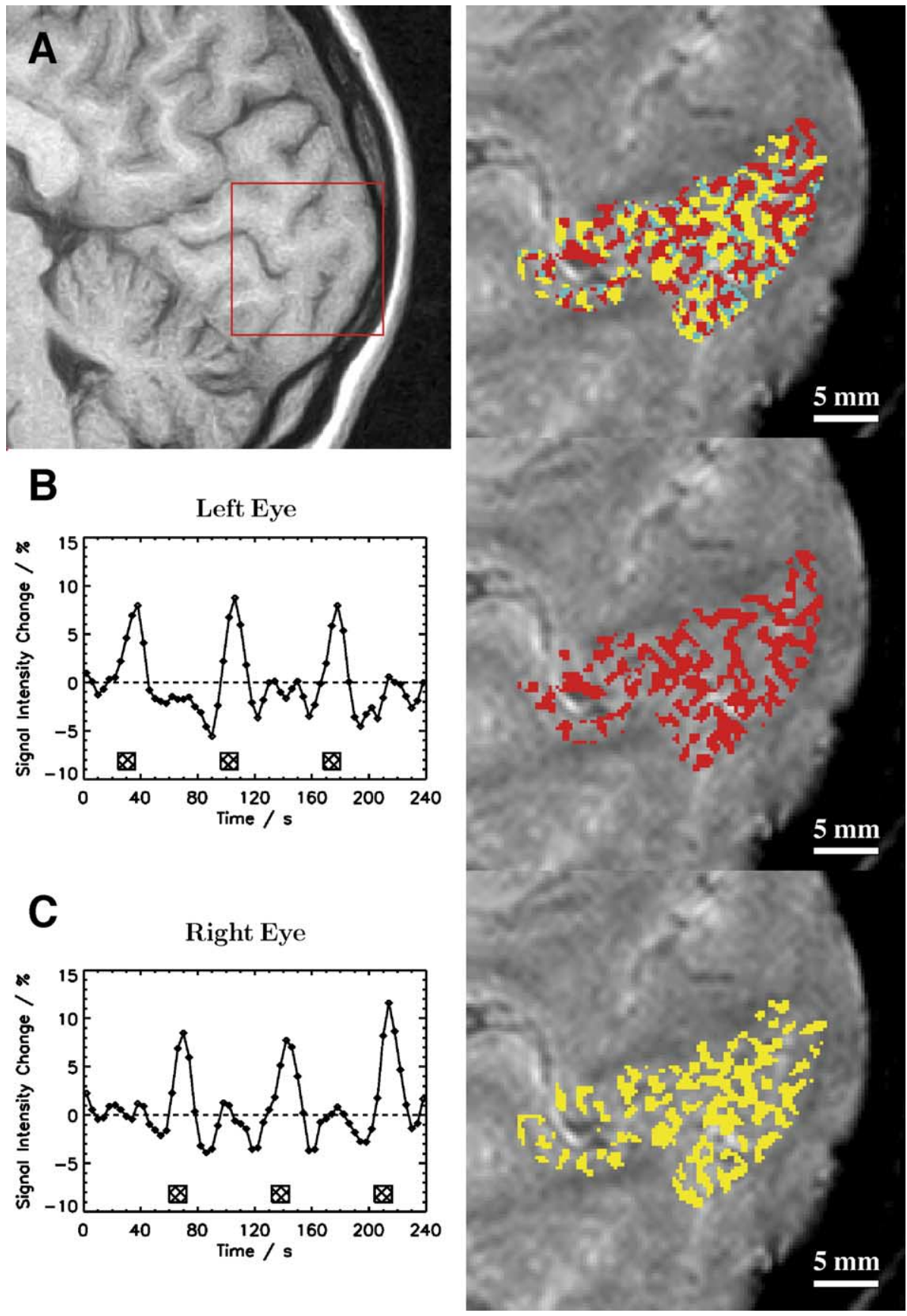

Fig. 9. Ocular dominance columns in human primary visual cortex. (A) Anatomic section covering the striate cortex and overlay of separate BOLD MRI activation maps obtained for selective (monocular) stimulation (checkerboard vs dark screen) of the left eye (red) and right eye (yellow). The absence of a substantial number of overlapping voxels (blue) confirms the identification of spatially segregated functional units predominantly coding for an individual eye (multi-echo FLASH, TR/TE $=105 / 40 \mathrm{~ms}, 15^{\circ}, 4 \mathrm{~s}$ measuring time, $0.25 \times 0.25 \times 4 \mathrm{~mm}^{3}$ interpolated from $0.5 \times 0.5 \times 4 \mathrm{~mm}{ }^{3}$ ). $(\mathrm{B}, \mathrm{C}$ ) The lower parts of the figure summarize (left) BOLD MRI signal intensity time courses and (right) corresponding activation maps of responses elicited by left and right eye stimulation, respectively. Reprinted from Ref. [85] with permission.

to minimize the risk of vascular signal contamination and false positive activations after intersubject averaging [90].

\subsection{Temporal resolution}

A current trend in functional MRI is the design of paradigms that improve the temporal efficiency of mapping studies and, perhaps more importantly, extend their applicability to established protocols in cognitive neuropsychology. Apart from exploiting the technical means for rapid data acquisition, pertinent attempts require not only sufficient functional contrast for brief cortical events but also the ability to distinguish respective BOLD MRI responses in time or space. In general, however, the latter is difficult to achieve as most mapping studies deal with brain regions exhibiting multi-functional encoding, 


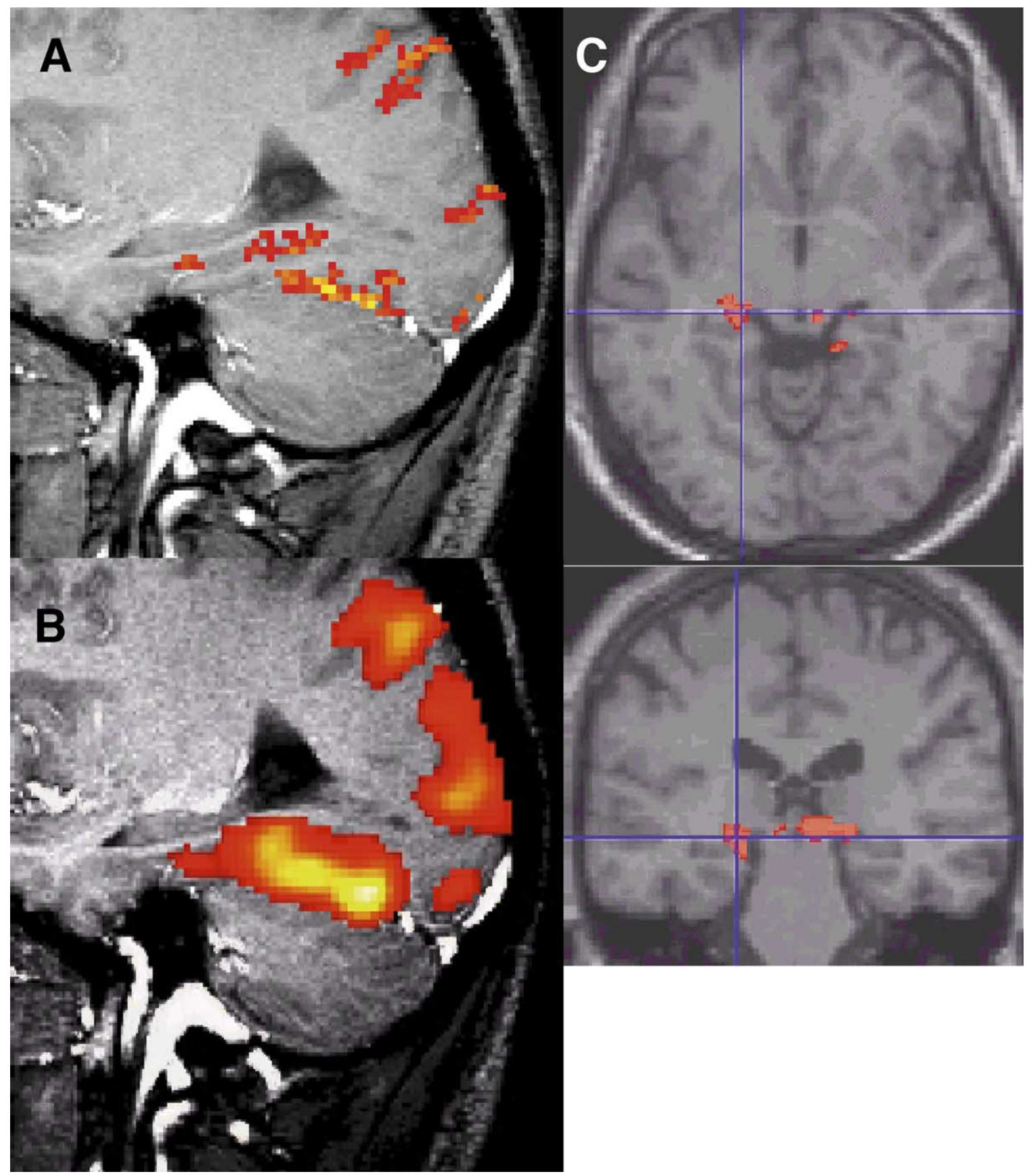

Fig. 10. Spatial filtering and intersubject averaging of BOLD MRI activations. (A) Oblique sagittal activation map of the right hippocampal formation (single subject, data analysis as in Fig. 2) demonstrating parahippocampal and visual activations during visual episodic memory encoding but no involvement of the hippocampus proper (sum of four neighboring echo-planar images acquired at $1 \mathrm{~mm}$ section thickness to reduce susceptibility artifacts). (B) A spatially filtered analysis of the same data using SPM99 (8 mm kernel) merges parahippocampal and visual activations. (C) A multi-subject conjunction analysis in approximate Tailarach space yields activations in the left hippocampus $\left(X=-20, Y=-28, Z=-6 ; T=2.41, p=5.1 \times 10^{-8}\right.$; axial and coronal SPM projections) not present in any of the single subject data. Adapted from Ref. [90] with permission.

that is, an overlap of subpopulations of neurons with different functional representations. Such coding principles already apply to visual and sensorimotor areas but are even more likely to occur for higher order cognitive processing. A typical example is the odd-ball paradigm designed to assess the brain's capability of detecting a novel event in a stream of rapidly presented stimuli. It is therefore necessary to examine the temporal evolution of BOLD MRI responses to rapidly repeated series of brief stimuli in more detail.

\subsubsection{Evolution of BOLD MRI responses}

In close agreement with the findings for longer stimuli, the BOLD MRI response profile elicited by a $1 \mathrm{~s}$ visual stimulus shown in Fig. 11 is characterized by an initial 

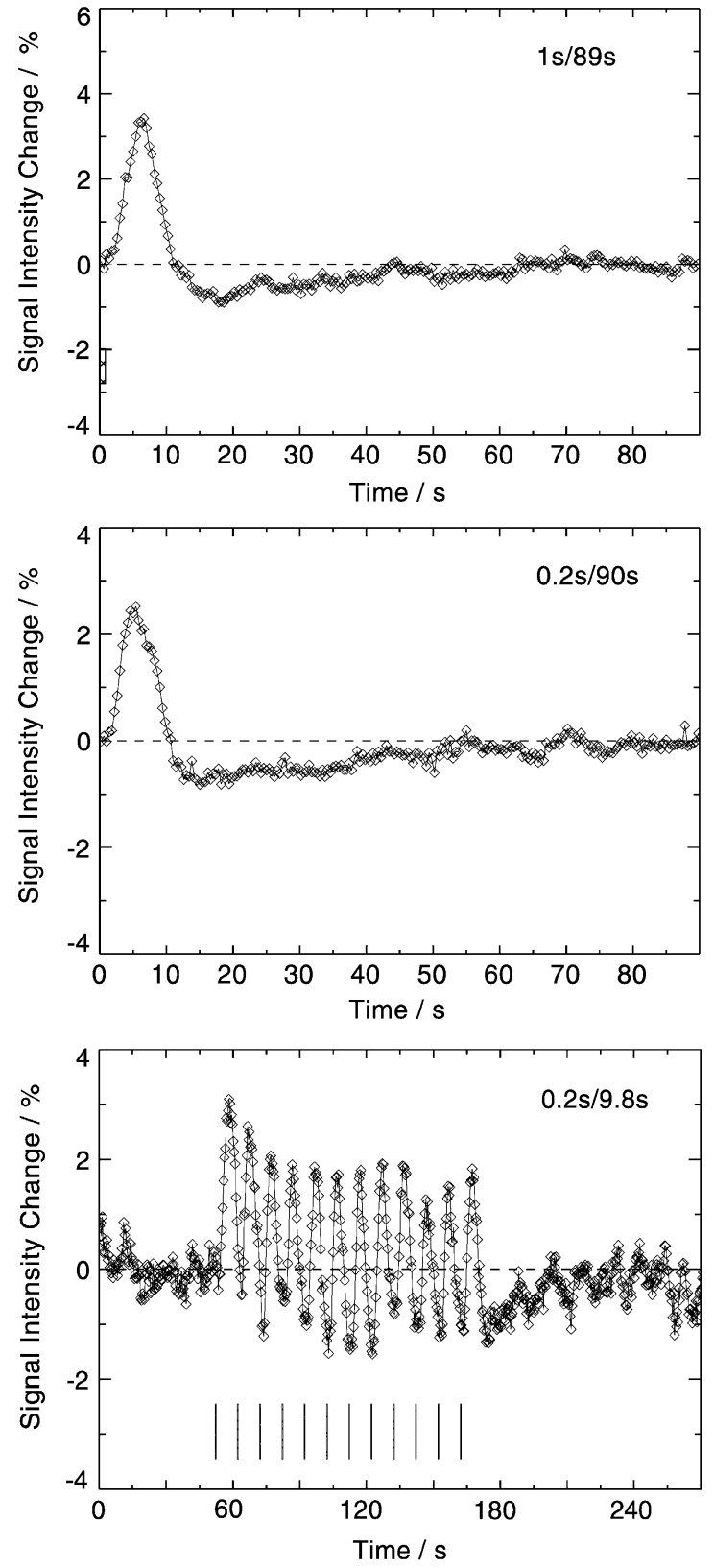

Fig. 11. Temporal evolution of BOLD MRI responses to brief visual stimuli. The data represent mean BOLD MRI signal intensity time courses averaged across subjects $(n=6)$ for (top) a 1 vs 89 s protocol, (middle) a 0.2 vs $90 \mathrm{~s}$ protocol, and (bottom) a repetitive $0.2 \mathrm{vs} 9.8 \mathrm{~s}$ protocol (checkerboard vs dark screen, parameters as in Fig. 1). Apart from a reduction in functional contrast, the BOLD MRI responses to both single and repetitive brief events resemble the same features as observed for longer stimuli (compare Fig. 1). Adapted from Refs. [92,93] with permission.

hemodynamic latency, a blood flow-related signal increase extending the duration of the actual stimulus, and a subsequent signal undershoot most likely reflecting increased blood volume [92]. In comparison with a 10-s presentation of the used checkerboard stimulus already shown in Fig. 1, the BOLD MRI response strengths of both the positive and negative signal deflection are reduced to about $3.5 \%$ (6 s after stimulus onset) and 1\% (15 s after stimulus onset), respectively. Extending these findings, the use of an even shorter 0.2-s stimulus results in a qualitatively identical pattern with a positive BOLD MRI response of about $2.5 \%$ at about $5 \mathrm{~s}$ after stimulus onset [93]. Because control periods of $60 \mathrm{~s}$ effectively decouple the physiological responses to individual stimuli, the mean time courses of Fig. 11 may be considered as reference response functions for the visual system thatnotwithstanding other linear or nonlinear interactionsunderlie net responses to more rapidly repeated protocols.

At least for brief visual stimuli of $1 \mathrm{~s}$ duration or less, the temporal evolution of the BOLD MRI response to repetitive stimulation may be qualitatively explained by an overlap of respective single response profiles. Fig. 11 demonstrates this behavior for a repetitive $0.2 \mathrm{~s}$ stimulation with a $9.8 \mathrm{~s}$ interstimulus interval. Under these circumstances it has to be asked whether the physiological history of cortical events alters the spatial activation pattern of the neural networks involved. Several studies support the view that temporal modulations of the BOLD MRI response do not affect the resulting activation maps and, therefore, will not hamper the future development of event-related mapping of activity changes, for example, see Ref. [92].

A further reduction of the interstimulus interval to less than the hemodynamic response time of about $5 \mathrm{~s}$, that is, from stimulus onset to maximum signal strength, markedly attenuates the functional contrast by precluding the full evolution of BOLD MRI responses to individual events. In order to distinguish responses with similar spatial encodings, it is therefore recommended to retain a sufficient decoupling of physiological responses in time and thereby ensure residual functional contrast. In the visual system, interstimulus intervals of $5 \mathrm{~s}$ duration are still able to map responses to individual $1 \mathrm{~s}$ stimuli in a repetitive protocol. Whether similar findings apply to other brain regions and stimuli remains to be established.

In a slightly different context, the observation of readily detectable MRI responses to very brief visual stimuli is of major relevance for the interpretation of slow fluctuations in the temporal 'noise' of BOLD MRI data sets that are obtained in the complete absence of a functional challenge or task performance. The data shown in Fig. 11 therefore provide experimental evidence for the assumption that brief cortical events or even spurious changes in brain activity may lead to physiological consequences with time constants of several tens of seconds. These findings lend further support for the use of low-frequency components in the BOLD MRI signal for deducing spatial patterns of functionally connected neural activity [94].

\subsubsection{EEG-correlated mapping of single events: epilepsy}

Because physiological brain activity is best reflected in the EEG signal, the aforementioned concept of studying ongoing neural activity in the resting awake brain is currently extended by combining EEG and fMRI recordings. 
The approach promises to benefit from exploiting complementary strengths in temporal and spatial resolution. Of course, simultaneous fMRI-EEG studies of normal or pathological brain activity require major efforts to obtain distortion-free EEG recordings during continuous functional MRI data acquisition, that is, in the presence of magnetic field gradient switches which induce electric currents in the EEG electrodes and cables. Based on recent technical progress, preliminary studies address the BOLD MRI responses to spontaneous EEG phenomena ranging from alpha rhythm and sleep patterns to epileptiform discharges and seizures $[95,96]$.

For example, the analysis of selected discrete EEG frequency bands revealed little positive correlation of localized brain activity with alpha power $(8-12 \mathrm{~Hz})$, but strong and widespread negative correlation in lateral frontal and parietal cortices that are known to support attentional processes. Power in the range of beta activity $(17-23 \mathrm{~Hz})$ was positively correlated with activity in retrosplenial, temporo-parietal, and dorsomedial prefrontal cortices which are involved whenever subjects engage in explicit perception or action [96]. Nevertheless, it should be pointed out that the transformation of EEG data into linear models suitable for voxel-based statistical hypothesis testing still depends on assumptions regarding the manner in which the generators of EEG phenomena may engender changes in the BOLD signal [95].

The ability of EEG-correlated fMRI to monitor transient changes in neural activity offers the potential to map pathological events. Such endeavors aim at the spatial localization or cerebral origin of electrophysiologically detected events. In particular, such applications should contribute to a source localization of aberrant electrical discharges in patients with epilepsy [97-102]. Fig. 12 shows a preliminary BOLD MRI recording of EEG-correlated interictal epileptic activity [97]. The patient suffered from a symptomatic, generalized epilepsy syndrome with up to 10 atypical absence seizures and a maximum of one generalized tonic-clonic seizure per day. Interictal EEG showed a diffusely slow background, a bi-frontotemporal theta-deltaslowing and generalized interictal polyspike and slow-wave complexes. Continuous EEG recordings with a commercially available MRI-compatible EEG system were performed during dynamic BOLD MRI using $2 \mathrm{~s}$ cycles of $0.5 \mathrm{~s}$ image acquisition and $1.5 \mathrm{~s}$ delay.

A post-examination derivation of an EEG reference function, which identified abnormalities for MRI analysis, revealed positive BOLD MRI responses with temporal characteristics similar to those obtained for functional challenges. Moreover, the corresponding map of 'activated' brain voxels yielded a contiguous area exhibiting a net decrease of the absolute concentration of deoxyhemoglobin in temporal correlation with the EEG abnormalities. The EEG reference function was shifted by $4 \mathrm{~s}$ relative to the onset of EEG spikes to account for the hemodynamic latency of the BOLD MRI signal as well as to eliminate
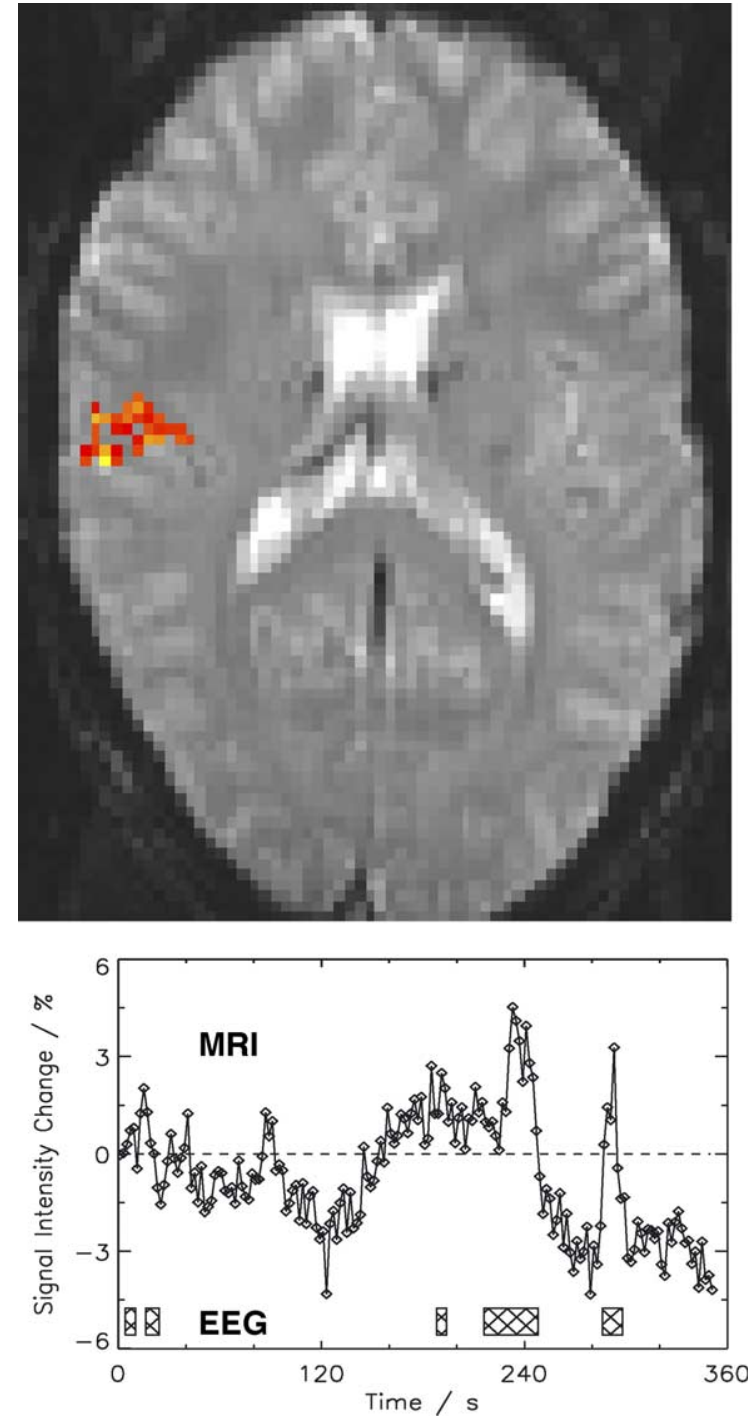

Fig. 12. Simultaneous EEG and BOLD MRI of a patient with a symptomatic generalized epilepsy syndrome. (Top) The activation map revealed a spatially congruent area of EEG-correlated epileptoform activity. (Bottom) The corresponding signal intensity time course represents BOLD MRI responses in relation to an EEG-derived reference function (boxes) which identified interictal EEG activity with polyspike and slow-wave complexes. Adapted from Ref. [97] with permission.

artifactual activations due to subject motions. The underlying rationale is the assumption that-if presentcorrelated motions occur as immediate movements during the first few seconds after the onset of spike and slow-wave complexes, whereas BOLD MRI responses to brief events exhibit minimum response times to maximum effect of about $5 \mathrm{~s}$ as demonstrated in Fig. 11.

The data lend support to the hypothesis that the hemodynamic alterations observed during interictal epileptiform activity rely on similar mechanisms as involved in processing sensory or cognitive input. An unresolved problem is the degree to which the EEG-correlated activations in the insular area correspond to the patient's primary epileptic zone. Although similar criticism also holds 
true for the generation of the EEG abnormalities, it remains an open question whether pertinent maps indicate true foci of epileptic activity or related processing of induced activity or both. Because epileptic activity tends to rapidly propagate to other cortical regions, it cannot be excluded that BOLD MRI responses occur not only in the primary epileptic zone but also in cortical projections of such regions as well as in other brain systems 'processing' various aspects of the actual event. Nevertheless, further developments of combined EEG and functional MRI recordings will open the way to study these questions in much greater detail and in a large number of patients.

\subsection{Technical problems}

Major technical problems of BOLD MRI acquisitions are caused by the presence of flow effects, stimulus-correlated motion, and structural susceptibility artifacts in brain regions affected by pronounced magnetic field inhomogeneities. With respect to flow, the early debate about a brainvein problem has been largely resolved because of progress in dealing with the most obvious problem, that is, direct macroscopic inflow effects [103]. Apart from using motion-rephasing gradient waveforms, an effective solution is to avoid any $\mathrm{T} 1$ saturation by combining

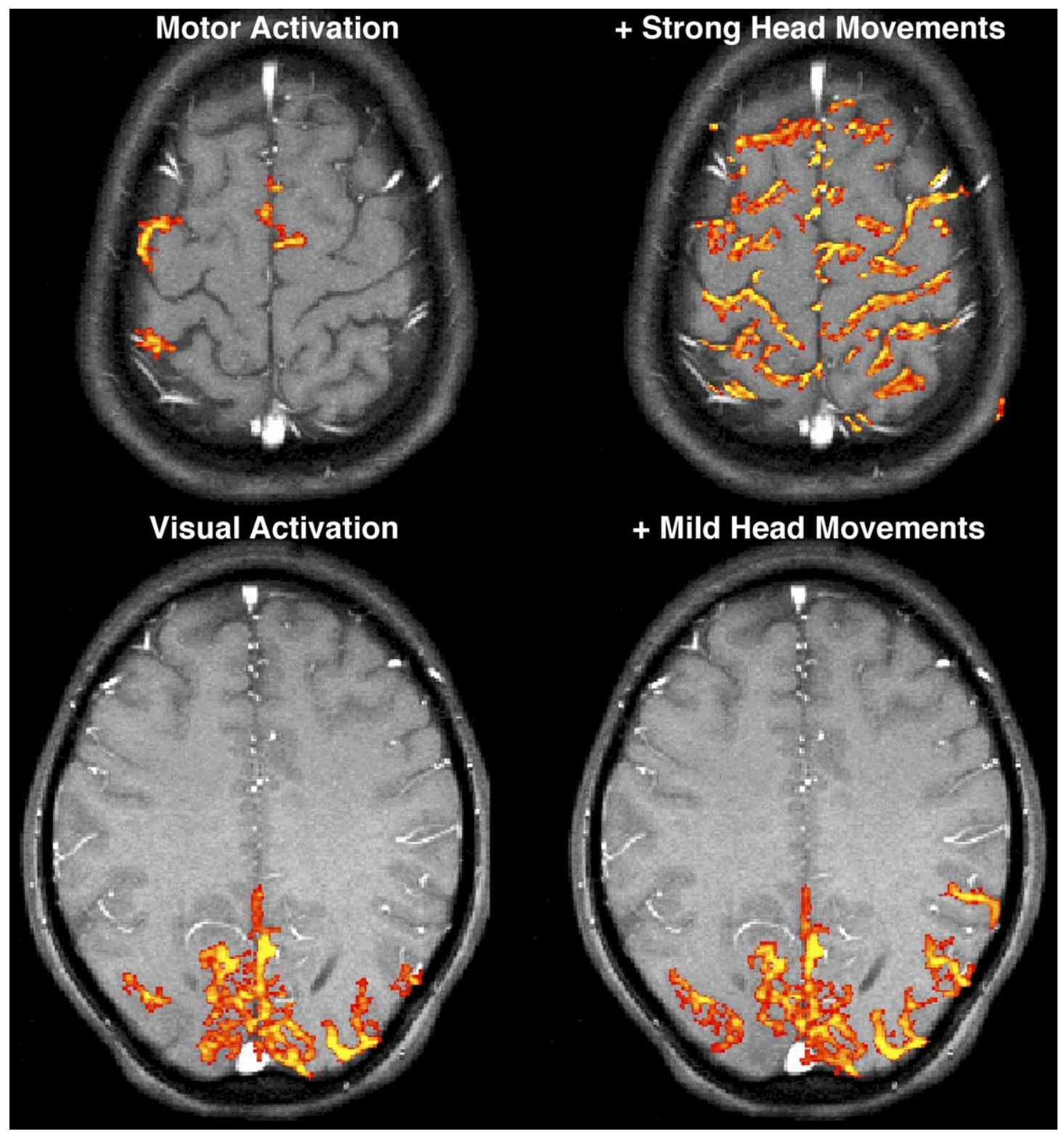

Fig. 13. Stimulus-correlated motion in BOLD MRI activation maps. (Top) Motor activation (finger tapping of the left hand) in the absence and presence of strong head movements as well as (bottom) visual activation (checkerboard vs dark screen) in the absence and presence of mild head movements. Whereas pronounced movements are easily detectable by enhancing all contrast borders in the original BOLD images (top right), mild stimulus-correlated motions may compromise the results by localized false positive activations (bottom right). 
the $\mathrm{T}^{*}$ sensitivity of the EPI (or FLASH) sequence with spin-density weighting: in general, this refers to a reduction of the flip angle below $90^{\circ}$ for repetition times of less than $5 \mathrm{~s}$ (at $1.5 \mathrm{~T})$.

\subsubsection{Correlated motions}

A serious threat to the reliability of an fMRI study is caused by the presence of stimulus-correlated motion [104] as shown in Fig. 13. The examples in the motor and visual system demonstrate that gross motions are readily identified by strong 'pseudo' activations at all contrast borders of the original BOLD-sensitive images. When eliminating T1 contrast, this mostly applies to CSF spaces which lead to a bright appearance of sulci. In contrast, small involuntary head motions in temporal correlation with the stimulus protocol pose an even more difficult problem as shown in the bottom row of Fig. 13. They may alter the noise structure and subsequent data analysis and, for example, unfavorably modulate the histogram of correlation coefficients in such a way as to generate localized false positive activations. Possible means proposed to alleviate the problem are post-acquisition techniques for motion correction by realignment of serial images and variations of the 'latency shift' during data analysis. As most of the practical problems originate from short-lasting movements in association with the presentation of a novel functional challenge, pertinent signal alterations may be separated from the delayed BOLD MRI response maximum by shifting the reference function with respect to stimulus onset.

\subsubsection{Susceptibility differences}

An almost unsurmountable problem results from the magnetic susceptibility difference between brain tissue and air-filled spaces such as the nasal cavity and auditory canal. Fig. 14 demonstrates marked signal losses and accompanying geometric distortions in EPI of the hippocampus despite the use of a $2 \times 2 \times 4 \mathrm{~mm}^{3}$ resolution normally considered to represent a very reasonable choice. In contrast to the microscopic (intravoxel) nature of the deoxyhemoglobinbased BOLD MRI contrast such macroscopic susceptibility artifacts not only depend on the echo time of a gradient-echo MRI sequence, but also on the voxel size: the smaller the voxel, the smaller the phase dispersion of individual spin isochromats within the volume, and therefore, the smaller the chance for mutual cancellation and signal void [105]. Consequently, a simple and effective solution to this problem stems from a reduction of the voxel size which for EPI is best accomplished by decreasing the section thickness [106]. The lower part of Fig. 14 shows improved EPI of the hippocampus obtained by adding four contiguous multislice EPI acquisitions at $2 \times 2 \mathrm{~mm}^{2}$ resolution and $1 \mathrm{~mm}$ section thickness. The prize to be paid for this improvement is a reduction of volume coverage by a factor of 4 .

Recently, similar comparisons addressed the reliability of functional mapping of the human amygdala [107]. As shown in Fig. 15, an EPI acquisition at $4 \times 4 \times 4 \mathrm{~mm}^{3}$ resolution

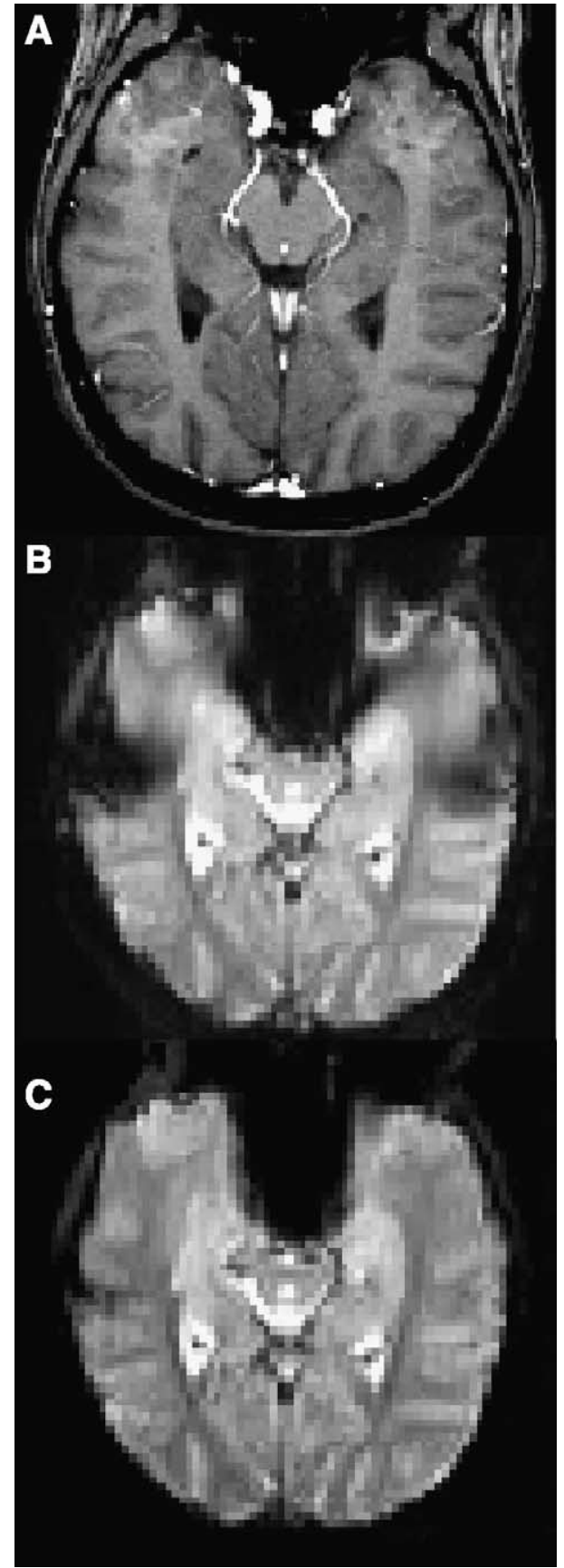

Fig. 14. Reduction of macroscopic susceptibility artifacts by BOLD MRI acquisitions of thin sections. (A) Anatomic reference image of the hippocampus ( $4 \mathrm{~mm}$ transverse section), (B) EPI at $2 \times 2 \times 4=16 \mathrm{~mm}^{3}$ resolution, and (C) EPI at $2 \times 2 \times 1=4 \mathrm{~mm}^{3}$ resolution (sum of four neighboring $1 \mathrm{~mm}$ sections as used for Fig. 10, other parameters as in Fig. 2). Adapted from Ref. [106] with permission.

yields a clearly visible intensity gradient which covers the amygdala even for the technically preferable coronal section orientation. Under such circumstances, stimulus-correlated motion may easily provoke false positive activations. When switching to EPI at $2 \times 2 \mathrm{~mm}^{2}$ resolution and $1 \mathrm{~mm}$ section thickness in Fig. $15 \mathrm{C}$, the situation markedly improves. However, the influence of the necessarily long 


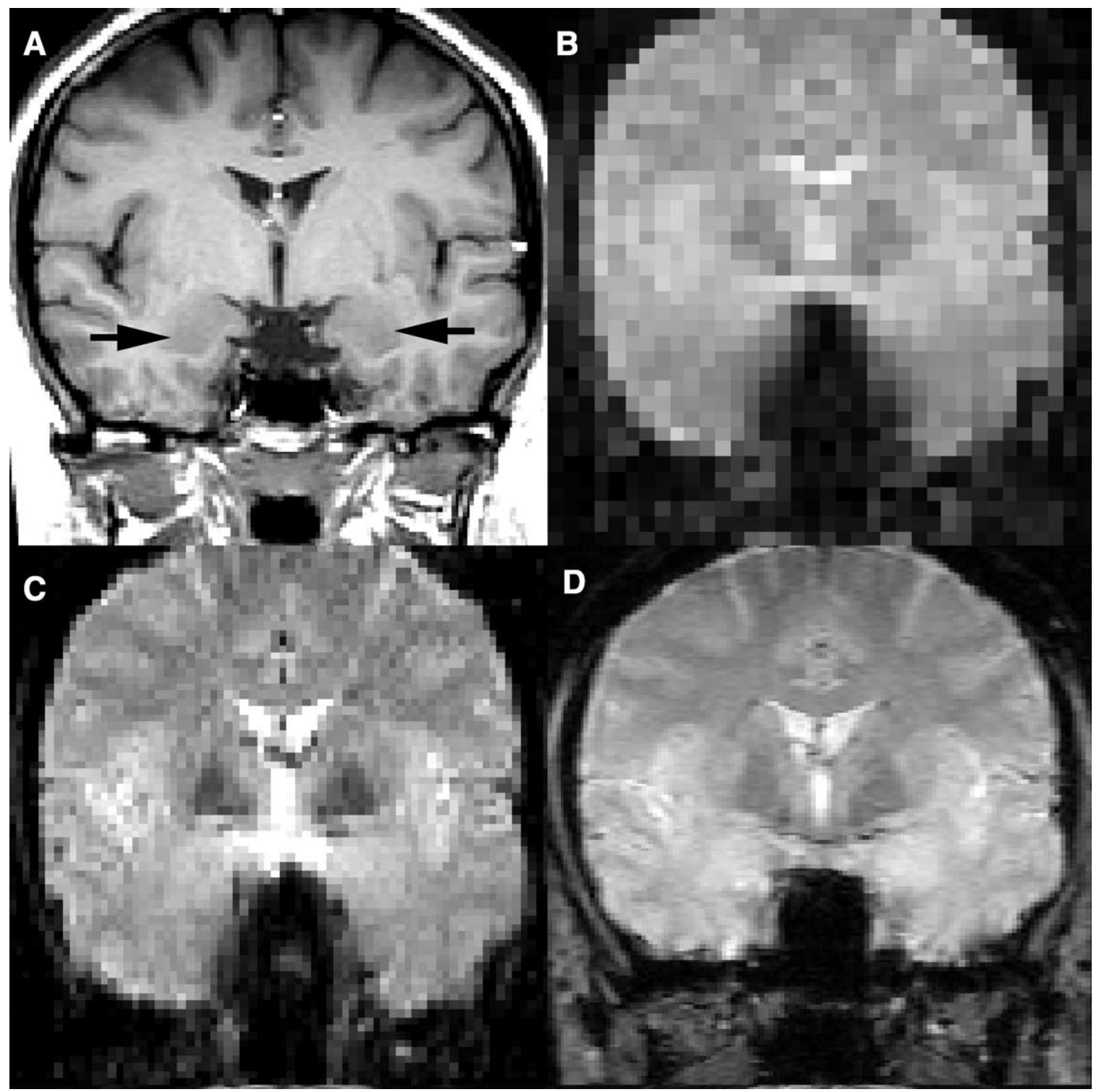

Fig. 15. Reduction of macroscopic susceptibility artifacts by BOLD MRI acquisitions of thin sections. (A) Anatomic reference image of the amygdala (arrows, $4 \mathrm{~mm}$ coronal section), (B) EPI at $4 \times 4 \times 4=64 \mathrm{~mm}^{3}$ resolution, (C) EPI at $2 \times 2 \times 1=4 \mathrm{~mm}^{3}$ resolution, and (D) FLASH (TE $\left.=30 \mathrm{~ms}\right)$ at $2 \times 1 \times 4=8 \mathrm{~mm}^{3}$ resolution. Adapted from Ref. [107] with permission.

echo time of the EPI sequence and the inherent sensitivity to differential resonance offset effects causes additional geometric distortions. This is not only seen relative to the anatomic reference scan in Fig. 15A, but also evident in comparison with a single-echo FLASH image in Fig. 15D. FLASH acquisitions further reduce the direct signal void, improve the homogenous signal within the amygdala, and match the anatomy in the reference image, whereas even $1 \mathrm{~mm}$ EPI sections suffer from a strong elongation of the brain along the direction of the phase-encoding blip gradient, that is, the vertical direction in Fig. 15C.

It turns out that EPI distortions, which are responsible for a lack of direct correspondence to the true anatomy, also occur in brain regions normally considered to be unaffected by strong susceptibility differences. This problem becomes another general concern when trying to correlate EPI-derived activation maps with brain anatomy as revealed by high-resolution T1- or T2-weighted MRI. The misregistration for functional mapping is demonstrated in Fig. 16 which compares the overlay of an EPI-derived BOLD MRI activation map of the motor cortex onto a T1-weighted anatomic scan with an overlay of the same map onto the original EPI acquisition. In the latter case, the finger movement is represented in the hand area of the primary motor cortex along the central sulcus, whereas the mismatch of the geometrically distorted activation map with the true anatomy yields an apparent posterior shift of the activation into the primary sensory cortex. It is therefore strongly recommended to overlay activation maps only onto anatomic images of similar geometric quality which for EPI normally restricts the choice to one of the original images acquired during functional mapping. 


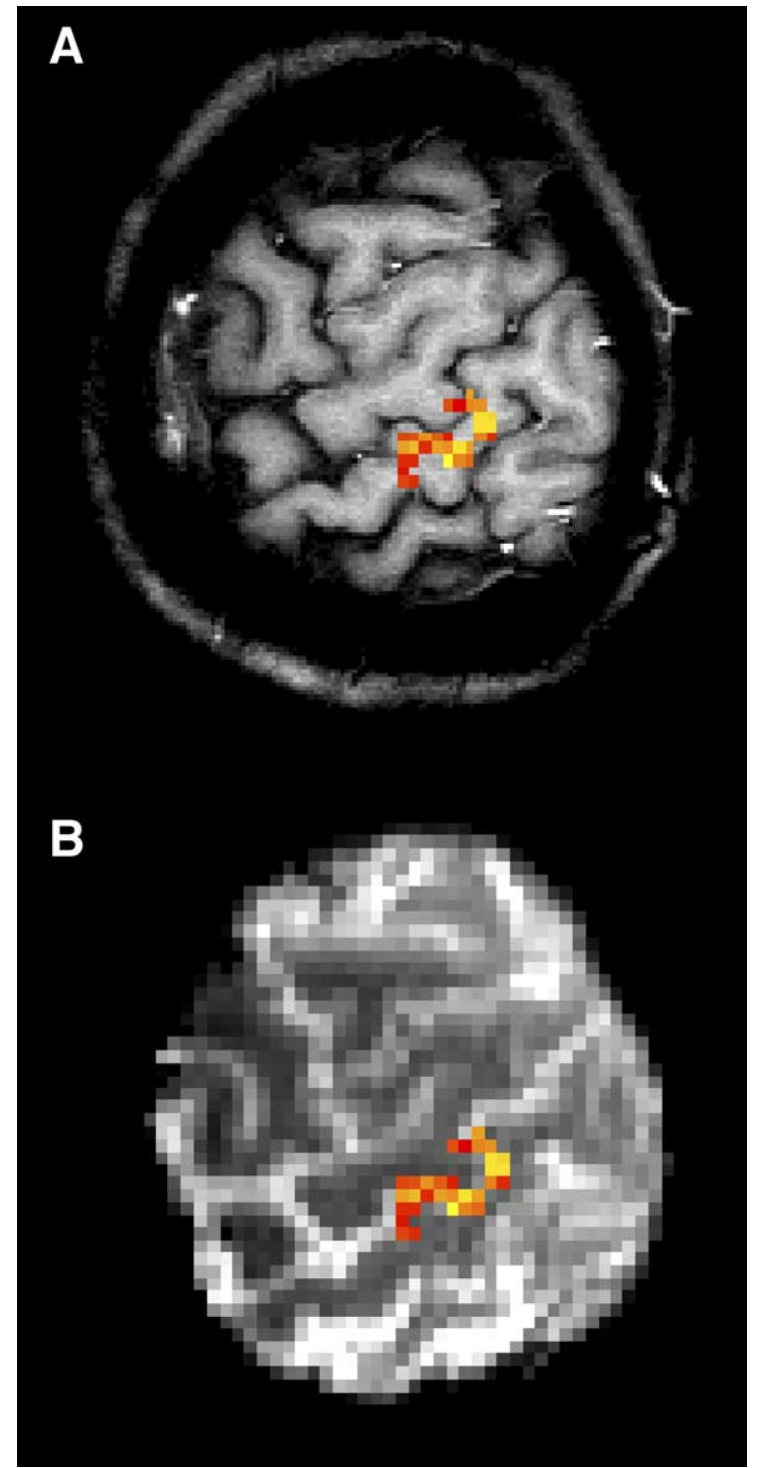

Fig. 16. Geometric distortions of EPI-derived BOLD MRI activation maps preclude an overlay with anatomic reference scans. EPI-derived activation map (finger tapping) superimposed (top) onto an anatomic reference image and (bottom) onto one of the originally acquired echo-planar images. Because of the EPI sensitivity to susceptibility effects, activations in the M1 hand area appear to be shifted onto the posterior post-central gyrus into the primary sensory cortex when using the spatially incongruous highresolution anatomic scan.

\subsection{Physiological problems: the neurovascular coupling}

The physiological response of vasomotor tone to vasodilatory stress from functional activation is one of the prime features of intact cerebral perfusion and a prerequisite for MRI of human brain function. More specifically, the approach relies on an intact neurovascular and neurometabolic coupling providing a stable relationship between neural activation and associated changes of the absolute deoxyhemoglobin concentration. Unfortunately, this translation of neural activity changes into BOLD MRI responses may be compromised by pharmacological and pathological modulations. Such manipulation may alter the individual subject's hemodynamic responsiveness to a functional challenge without affecting the neural function. Examples are the use of drugs with a vasoactive component, functional mapping studies under anesthesia [108], and cerebrovascular disease affecting the brain's autoregulatory reserve capacity. Moreover, cortical deactivation has been observed due to visual stimulation in human slow-wave sleep [109] and modulations of the BOLD MRI response were found both in young children in the age group with the highest synaptic density [110] as well as in the elderly yielding age-related reductions of the functional contrast in response to visual stimulation [111] or motor performance [112].

\subsubsection{Pharmacological modulations}

Although pharmacological depression and stimulation using psychotropic drugs such as diazepam and metamphetamine have also been reported to elicit distinct BOLD MRI changes [113], quantitatively more pronounced drug-induced changes in vasomotor tone and BOLD MRI signal strength are observed for acetazolamide [114] as vasodilator and aminophylline [115] as vasoconstrictor. These results may have clinical impact in the application of functional MRI to patients taking pertinent medication and are also of scientific interest when aiming at a better understanding of differing mechanisms contributing to the BOLD MRI contrast. Fig. 17 summarizes the results obtained for the potent vasoconstrictor indomethacin known to decrease cerebral blood flow without affecting the cerebral metabolic rate of oxygen [116]. Similar to acetylsalicylate, indomethacin acts as a strong inhibitor of prostaglandine synthesis which is the reason for its widespread use for antiinflammatory and analgesic purposes.

In contrast to isotonic saline and acetylsalicylate as control conditions, indomethacin causes an immediate signal decrease of the resting state BOLD MRI signal within the 2-min application period. The effect ranges from 2.1 to $2.6 \%$ in cortical and subcortical gray matter shown in the top row of Fig. 17 and $1.2 \%$ in white matter. It reflects an increase of the absolute deoxyhemoglobin concentration throughout the cerebral vasculature as an indirect measure of vasoconstriction and ensuing blood flow decrease. With respect to visual activation neither acetylsalicylate nor saline reveal alterations of the BOLD MRI signal pattern or functional contrast. In contrast, as shown in the middle and bottom parts of Fig. 17, indomethacin causes a mild reduction of the initial BOLD MRI signal strength to about $85 \%$ followed by a marked decrease of response magnitude to only $66 \%$ of the pre-drug functional contrast for a repetitive protocol and $47 \%$ for sustained stimulation. Thus, despite pronounced vasoconstriction, the initial BOLD MRI response suggests an intact physiological response to neurostimulation in terms of upregulated blood flow. The observation of a subsequent attenuation of the response strength during both sustained and repetitive 

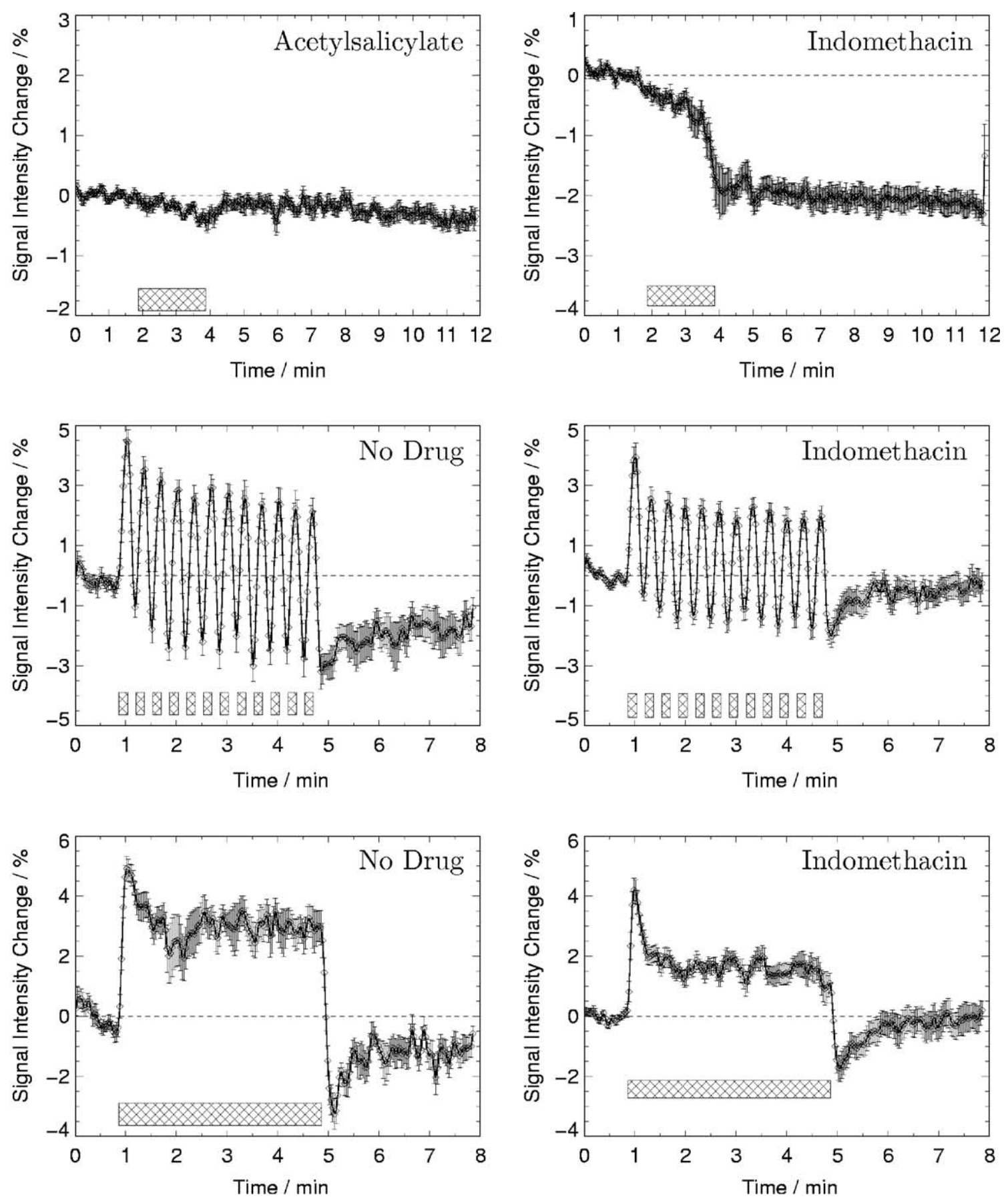

Fig. 17. Modulation of BOLD MRI activations by indomethacin. (Top) In comparison with acetylsalicylate, administration of vasoconstrictive indomethacin ( $2 \mathrm{~min}$ ) reduces blood flow, so that the concomitant increase in deoxyhemoglobin decreases the resting state BOLD MRI signal $(n=10)$. (Middle) Relative to isotonic saline (no drug), administration of indomethacin reduces the functional contrast of subsequent BOLD MRI responses to repetitive visual stimulation (checkerboard vs gray screen, $n=10$ ) and (bottom) attenuates the response to sustained visual activation $(n=8)$. Adapted from Ref. [116] with permission.

stimulation suggests the vasoconstrictive action of indomethacin and the vasodilative response to a functional challenge to be governed by multiple mechanisms.

\subsubsection{Pathological alterations}

The ability to rapidly adapt the vasomotor tone to functional activation or pharmacological manipulation presents a considerable autoregulatory reserve capacity of the brain. Hemodynamic compromise in cerebrovascular disease stresses this reserve capacity as shown for the case of an asymptomatic patient with unilateral occlusion of the internal carotid artery (ICA) [117] in Fig. 18. Evaluating responses to additional vasodilatory stress as, for example, after application of the carbonic anhydrase inhibitor acetazolamide can assess the effect. While resting BOLD MRI signal intensities reveal no left-right 

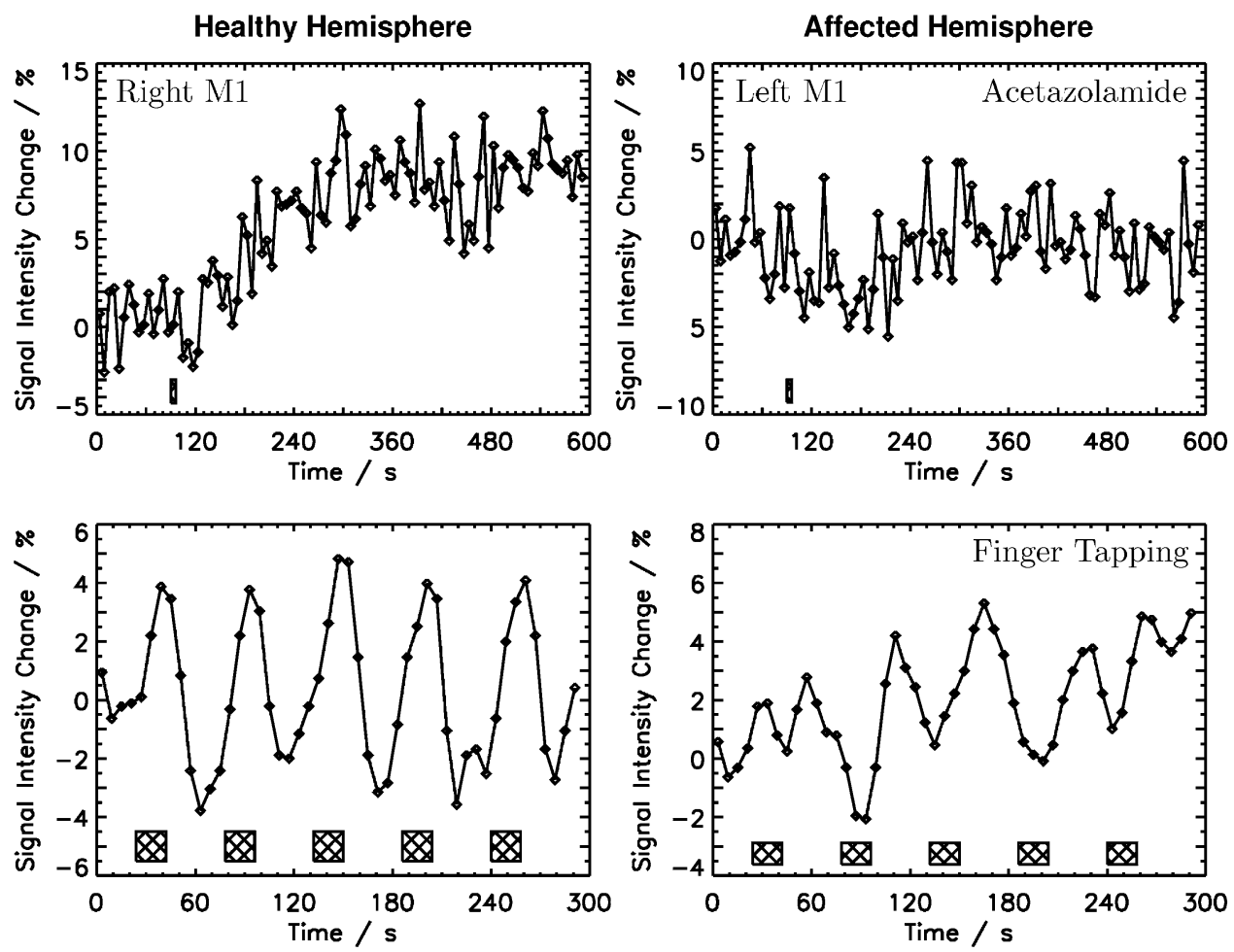

Fig. 18. Modulation of BOLD MRI activations by cerebrovascular disease. (Top) BOLD MRI responses to vasodilating acetazolamide (1.0 g bolus) in an asymptomatic patient with left-hemispheric occlusion of the internal carotid artery reveal a normal increase in blood flow and concomitant reduction in deoxyhemoglobin in the unaffected right hemisphere. In contrast, the lack of a response in the affected left sensorimotor cortex suggests an exhausted reserve capacity maintaining residual cerebral perfusion. (Bottom) Similarly, the normal BOLD MRI responses to finger tapping of the left hand in right M1 are opposed to mainly negative signal changes elicited by the right hand in the affected left M1. The observation suggests that the absence of a change in blood flow unmasks the contribution of elevated deoxyhemoglobin due to upregulated oxygen metabolism during a functional challenge. Adapted from Ref. [117] with permission.

asymmetries (not shown), acetazolamide administration ( $1.0 \mathrm{~g}$ bolus) induces an overall rise in signal intensity in the normally perfused right hemisphere in accordance with results in healthy subjects [114]. In contrast, the hemisphere ipsilateral to the occluded ICA shows a widespread unresponsiveness to vasodilatory stress unmasking a pathological compromise of autoregulation. Because oxygen metabolism remains stable under acetazolamide [118] the BOLD MRI response represents a direct measure of blood flow, so that its absence indicates a failure to further stimulate blood flow. This finding offers new means to assess and even to map the impairment of the autoregulatory reserve capacity in patients with carotid occlusive disease.

The situation of an exhausted reserve capacity, which maintains residual blood flow despite low arterial pressure, poses a severe complication for mapping brain function. As shown in the bottom part of Fig. 18, finger tapping of the left hand yields a normal BOLD MRI response in the unaffected right sensorimotor cortex hand area. Conversely, the corrupted pattern observed for responses to the right hand in the affected left hemisphere appears to be inverted yielding mainly BOLD MRI signal decreases in temporal coincidence with finger tapping. Under the assumption of unchanged blood flow (and volume) as indicated by the absence of an acetazolamide response, the neurofunctional BOLD MRI response most likely reflects the increased deoxyhemoglobin concentration caused by an upregulation of oxygen metabolism during neural processing. Recent investigations indeed support the notion of a significant increase in oxygen consumption and concomitant production of deoxyhemoglobin during brain activation [43] which adds to the BOLD MRI response as a negative signal contribution.

\subsection{Paradigm design: a persistent challenge}

Beyond an understanding of the methodology, the proper design of the paradigm turns out to be of utmost importance. This particularly applies to the reliability of an increasing number of functional MRI studies addressing higher order cognitive and emotional processing. In fact, many literature examples already raise serious doubts whether the naive adaptation of a neuropsychological test protocol achieves both a complete and exclusive translation of the original scientific question into an MRI-compatible paradigm. While caution is generally more accepted when studying language, memory, and learning, also primary sensory systems such as vision and sensorimotor control present with similar problems. The existence of multiple functional 
coding principles in the visual system is partly demonstrated in Figs. 3, 4 and 9. In addition, visual perception is strongly regulated by 'top-down' processes which interfere with specific aspects of the complex visual input resulting from 'bottom-up' seeing. For example, a most prominent area of research is the modulation of visual perception by attention [119].

This section contains two simple examples which serve to illustrate the need for a careful design of a specific paradigm as well as for a suitable strategy for data analysis. The first case deals with stereoscopic depth perception and the observation of transient vs sustained BOLD MRI responses [120], while the second study addresses a long-term controversial issue in motor control, namely the fine-scale somatotopic organization of the primary motor cortex hand area [121].

\subsubsection{Stereoscopy}

The visual system of humans and many animals is able to recover the $3 \mathrm{D}$ shape of objects and the spatial layout of the environment from the two-dimensional (2D) projections of the visual world onto their retinas. While many sources of information such as shading, texture gradients, motion, occlusions, and object size contribute to this ability, a central element of depth information is binocular disparity, that is, the slight differences between the images in the left and right eye caused by their different viewing angle.

\section{Single Image Stereogram}

Self - Controlled Switches
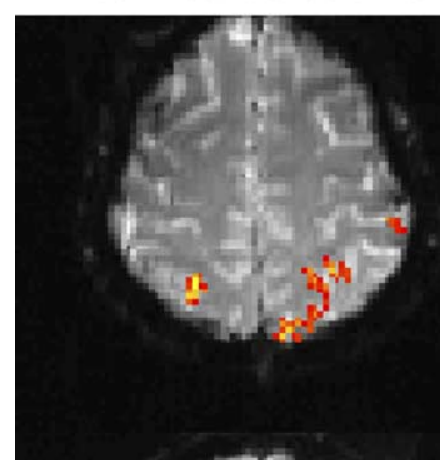

(1)
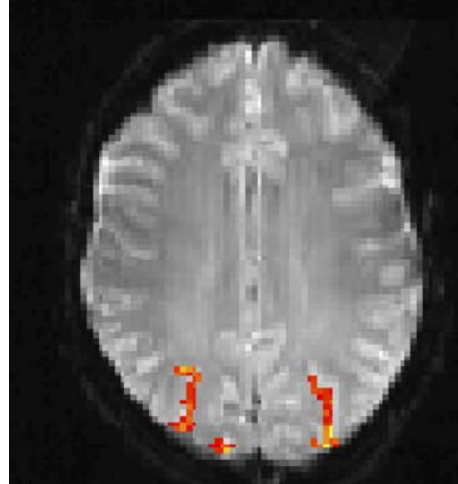

AB - AA

\section{D Visual Processing}

AA - Gray Screen
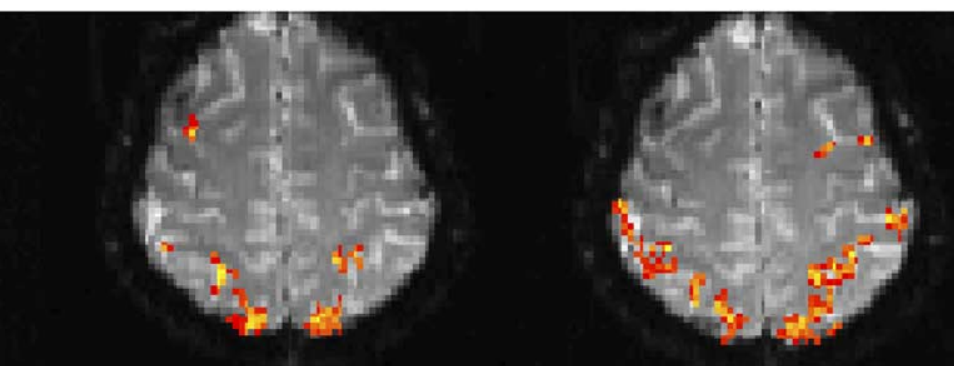

Antry
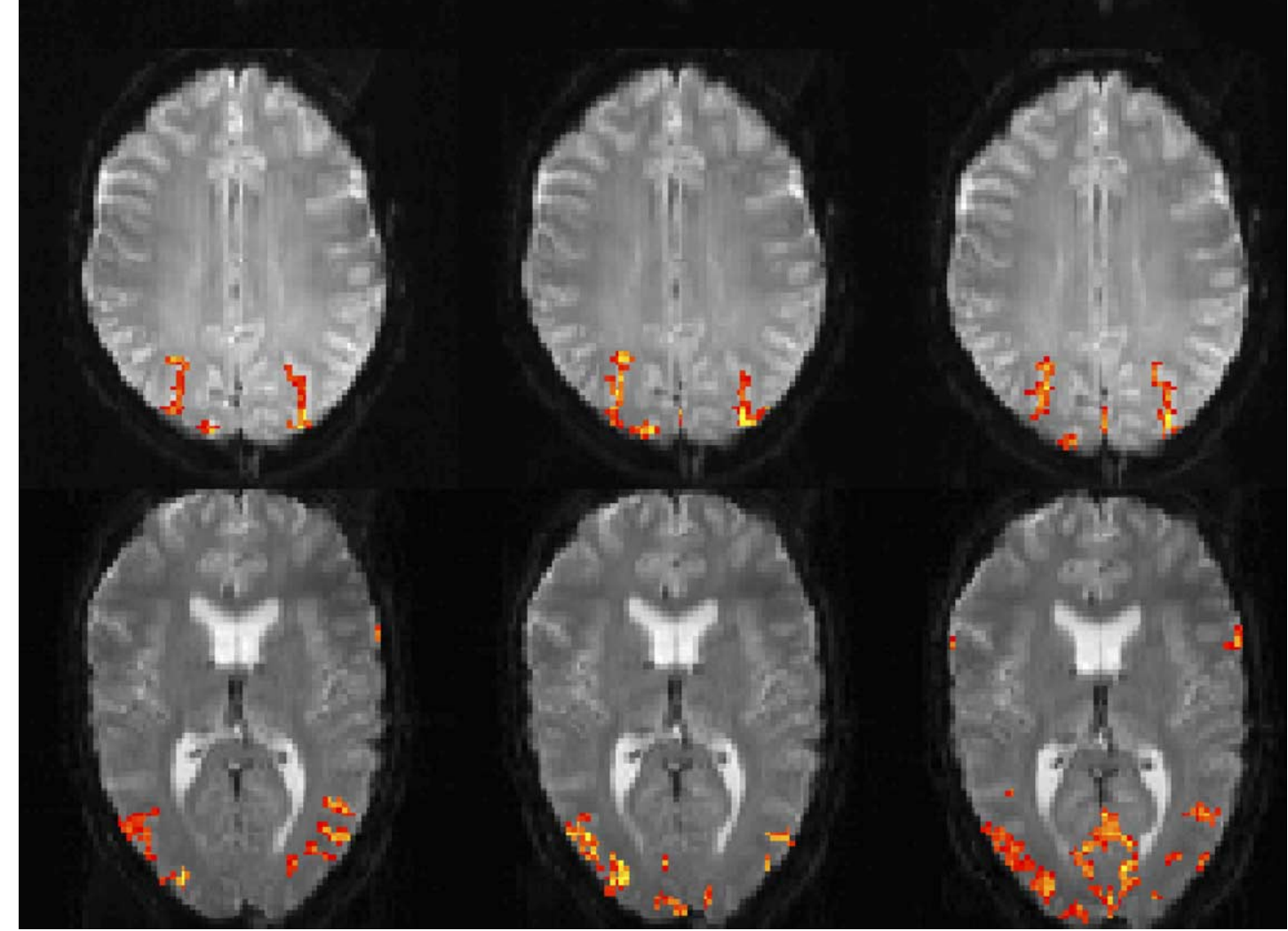

Fig. 19. Functional MRI of stereoscopic depth perception. Selected activations in (top) parietal, (middle) parieto-occipital, and (bottom) occipital cortex (single subject, 3/16 sections, other parameters as in Fig. 2) for (left) a single-image stereogram (falling coins under slightly different view angles which allowed experienced subjects to perform self-controlled switches between 2D viewing and depth perception), (middle) a pair of stereoscopic images A and B (views from the Eiffel tower at slightly different angles presented to the right and left eye, respectively) contrasted with a corresponding $2 \mathrm{D}$ perception in an $\mathrm{AB}$ vs $\mathrm{AA}$ block design, and (right) 2D visual processing (AA vs gray screen). Reprinted from Ref. [120] with permission. 
The traditional approach to precisely manipulate stereoscopic disparity for experimental purposes is the use of stereoscopic images. In such a design subjects are presented with different images to their left and right eye. A typical example is a pair of images of the same object but with slightly different view angles, denoted A and B, which together cause a 3D percept. In order to dissociate brain activations in relation to stereoscopic depth perception from the processing of other stimulus features, the subjects also view two identical images as controls, that is, either $\mathrm{A}$ and $\mathrm{A}$ (AA) or B and B (BB). The residual physical difference of the two stimuli in an $\mathrm{AB}$ vs $\mathrm{AA}$ block design may be completely avoided by the use of single-image stereograms. While viewing such a stimulus, the embedded binocular disparity can create a perception of depth, so that trained subjects are able to switch between 2D and 3D perception without any change of visual content. Accordingly, the experimental design for functional neuroimaging relates to event-related mapping of the self-controlled switches between the two perceptual states.

Fig. 19 compares selected activation maps of a single subject obtained for viewing (left) a single-image stereogram, (middle) a pair of stereoscopic images contrasted with two identical 2D images, and (right) a control paradigm which tested for the $2 \mathrm{D}$ visual features of the $\mathrm{AA}$ or $\mathrm{BB}$ images when contrasted with a gray screen [120]. Confirming previous findings, this latter paradigm illustrates that the human visual system extends over major parts of the occipital and parietal cortex yielding strong and widespread activations for $2 \mathrm{D}$ visual processing. The results for single-image stereograms revealed a distributed network of cortical areas embedded within the visual pathways. Quantitatively, it included about one quarter of the cortical surface activated by $2 \mathrm{D}$ visual stimulation. Three-dimensional perception recruited mostly neural populations in higher order visual areas: whereas about $40 \%(90 \%)$ of the visually activated locations along the intraparietal sulcus were also activated by $3 \mathrm{D}$ perception based on single-image stereograms (stereoscopic images), only $10 \%$ such overlap was found in striate cortex.

Surprisingly, no activations were found for the AA vs BB paradigm despite the fact that all subjects reported as having recognized the changes of the view angle at the start of each block presentation. Because this suggests a transient adaptation to a new visual percept, the occurrence of corresponding brain activity may have escaped the data analysis when asking for a sustained response to the blockdesign protocol. A more suitable search for activations at the onset of each new image presentation using a short-lasting reference function ( $6 \mathrm{~s}$ duration) yielded activations within the intraparietal sulcus in $4 / 5$ subjects, which may have contributed to the findings for stereoscopic images. As an example, Fig. 20 compares the activations obtained for a new percept (transient response to AA vs BB) with those for stereoscopic images (sustained response to $\mathrm{AB}$ vs $\mathrm{AA}$ ) and visual processing (sustained response to AA vs gray). Based on the overlap, it is tempting to speculate that the recognition of and attention to an altered view serves to initiate depth processing by testing for binocular disparity in order to generate a new 3D perception [120].

\subsubsection{Finger somatotopy}

Complementing the discussion of Section 3.6.1, recent studies of the somatotopic arrangement of individual fingers in the human primary motor cortex clarified previously contradictory results when considering subtle though qualitatively important differences in the experimental design (or data analysis). Although a gross somatotopy of movement representations in M1 has been well established for major body parts, the degree of fine-scale somatotopy remained an unresolved and controversial issue. Early MRI
Transient Response

$A A-B B$
Stereoscopic Images

$A B-A A$
2D Visual Processing

AA - Gray Screen

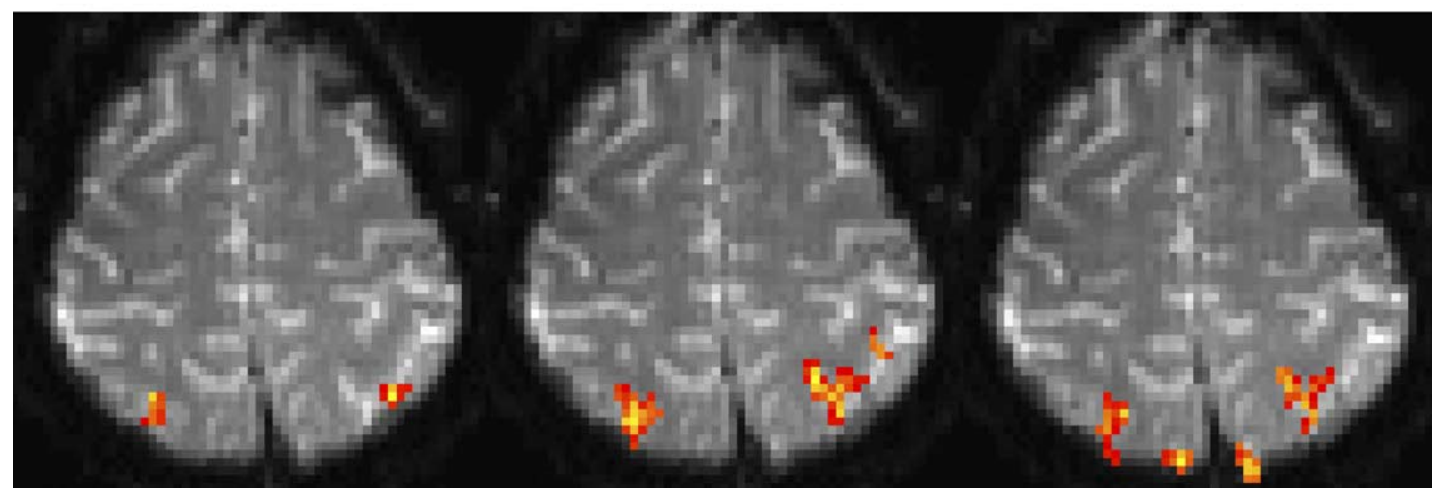

Fig. 20. Functional MRI of transient responses to the recognition of a new visual percept. (Left) Whereas a change of view angle (AA vs BB) does not lead to any sustained activation due to the presentation of the AA or BB stimulus (not shown), the initial recognition of a new visual percept gives rise to a transient response in the intraparietal sulcus (parameters as in Fig. 19). These activations overlap with activations caused by sustained responses to (middle) stereoscopic depth perception (AB vs AA) and (right) 2D visual processing (AA vs gray screen). Adapted from Ref. [120] with permission. 
studies either supported the concept of a distributed organization of finger representations with substantial overlap [122] or, at least in part, provided evidence for a somatotopic arrangement within the hand area [123]. It turns out that this situation mainly reflects the use of motor paradigms which biased the functional responses toward either spatially distributed or segregated representations.

The problem is shown in Fig. 21 comparing individual digit representations of the thumb and little finger in human M1 [123]. Respective activations were obtained for a paradigm contrasting a finger movement with (top) motor rest or (bottom) another finger movement. Whereas finger movement vs rest asks for all representations of neural activity involved in performing the requested motor task, the differential paradigm of two finger movements highlights predominant activations in the presence of spatial overlap. Fig. 21 indicates both a complete coverage of the motor hand area by individual finger representations and a spatially distinct arrangement of predominant finger representations with the thumb at the lateral end and the little finger in a more medial portion of the hand area.

The hypothesis of two complementing encoding principles, that is, overlap as well as spatially ordered functional predominance of finger representations, was recently re-examined by combining direct and differential mapping of individual finger movements for all five digits of the dominant hand [121]. In order to facilitate a visual inspection of the large number of activation maps, the data of individual subjects were projected onto a flattened part of the left-hemispheric M1 hand area without its complex cortical convolution. As shown in Fig. 22 the procedure involves a segmentation of anatomic 3D MRI data at the gray-white matter boundary and a subsequent inflation of the left hemisphere and magnification of the M1 hand area along the central sulcus.

Fig. 23 summarizes the key results of the mapping study. First of all, a direct paradigm contrasting finger movements with motor rest reveals considerable overlap of single digit representations covering large portions of the M1 hand area (top). These observations do not support the prevalence of a classic somatotopy with spatially separated and monotonously ordered finger representations that refer to independent finger-specific subdivisions of the M1 hand area. Instead such representations are widely distributed and their spatial extent causes considerable overlap. On the other hand, differential mapping opposes a single finger movement with that of another finger and thereby achieves smaller activation clusters with significantly reduced
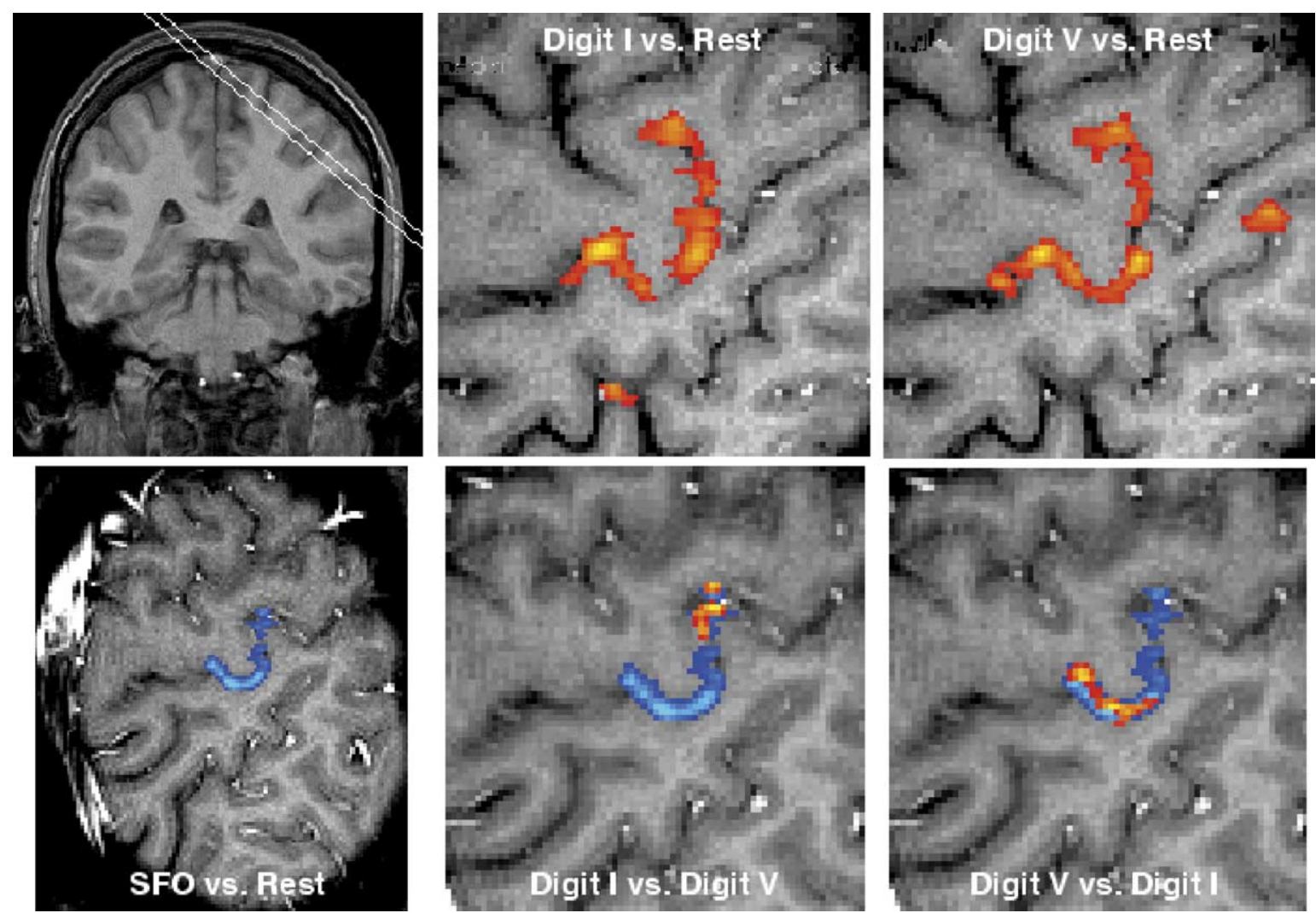

Fig. 21. Functional MRI of finger representations in human primary motor cortex. (Left) Section orientation and corresponding activation map of the lefthemispheric primary motor cortex obtained for a right-hand motor task (SFO: sequential finger-to-thumb opposition, blue). (Middle and right) Depending on the chosen paradigm design, activations (red) obtained for movements of the (middle) thumb or (right) little finger reveal either (top) a full coverage of the hand area and therefore complete overlap when contrasted with motor rest or (bottom) spatially segregated activation foci when contrasted with a different finger movement. Reprinted from Ref. [123] with permission. 


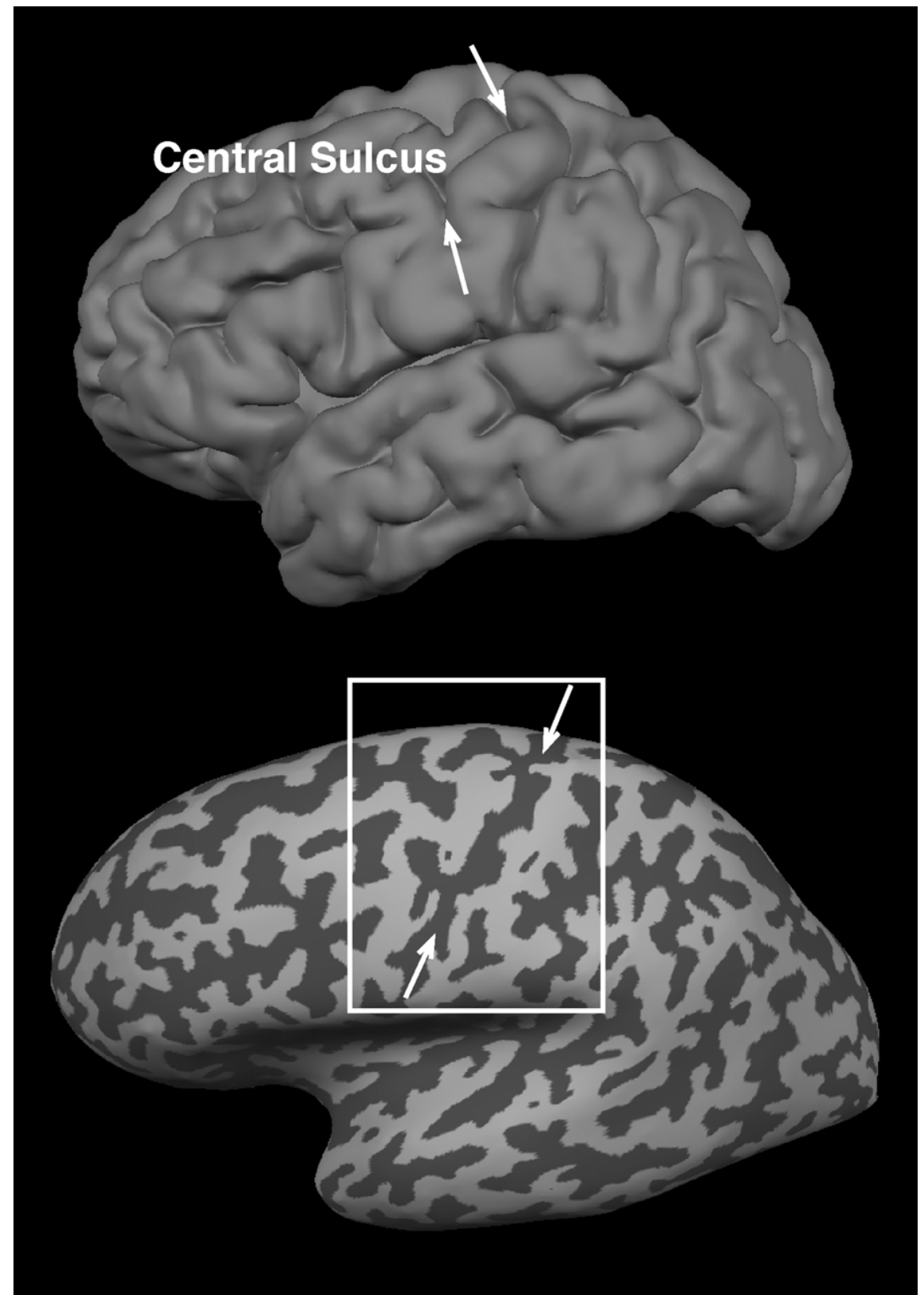

Fig. 22. Reconstructions of the (top) cortical surface and (bottom) inflated gray matter from a 3D MRI anatomic data set. Arrows indicate the approximate motor hand area along the left-hemispheric central sulcus. Reprinted from Ref. [121] with permission.

overlap (bottom). Moreover, the foci of differential representations are arranged in a somatotopic order extending from a lateral-anterior-inferior location representative of digit D1 (thumb) to a medial-posteriorsuperior position for D5 (little finger).

Combining the apparently diverging results of both paradigms, the human M1 hand area presents as a physiologically synergetic and anatomically interconnected area, with fine-scale somatotopy implemented as a relative quantitative predominance of individual finger representations sharing common areas. This redefined concept of functional somatotopy reconciles previous contradictory views by mapping the residual differential representations of two (or more) finger movements after 'subtracting' shared neural populations. In a more general sense, it accommodates both coordinated common movements and independent individualized movements and thus fits the emerging model of a functional organization of M1 comprising convergence, divergence, and horizontal connections [124].

\section{Conclusion}

After the first decade of deoxyhemoglobin-sensitive MRI sequences which yield dynamic BOLD MRI contrast in response to a functional challenge, the potential for 


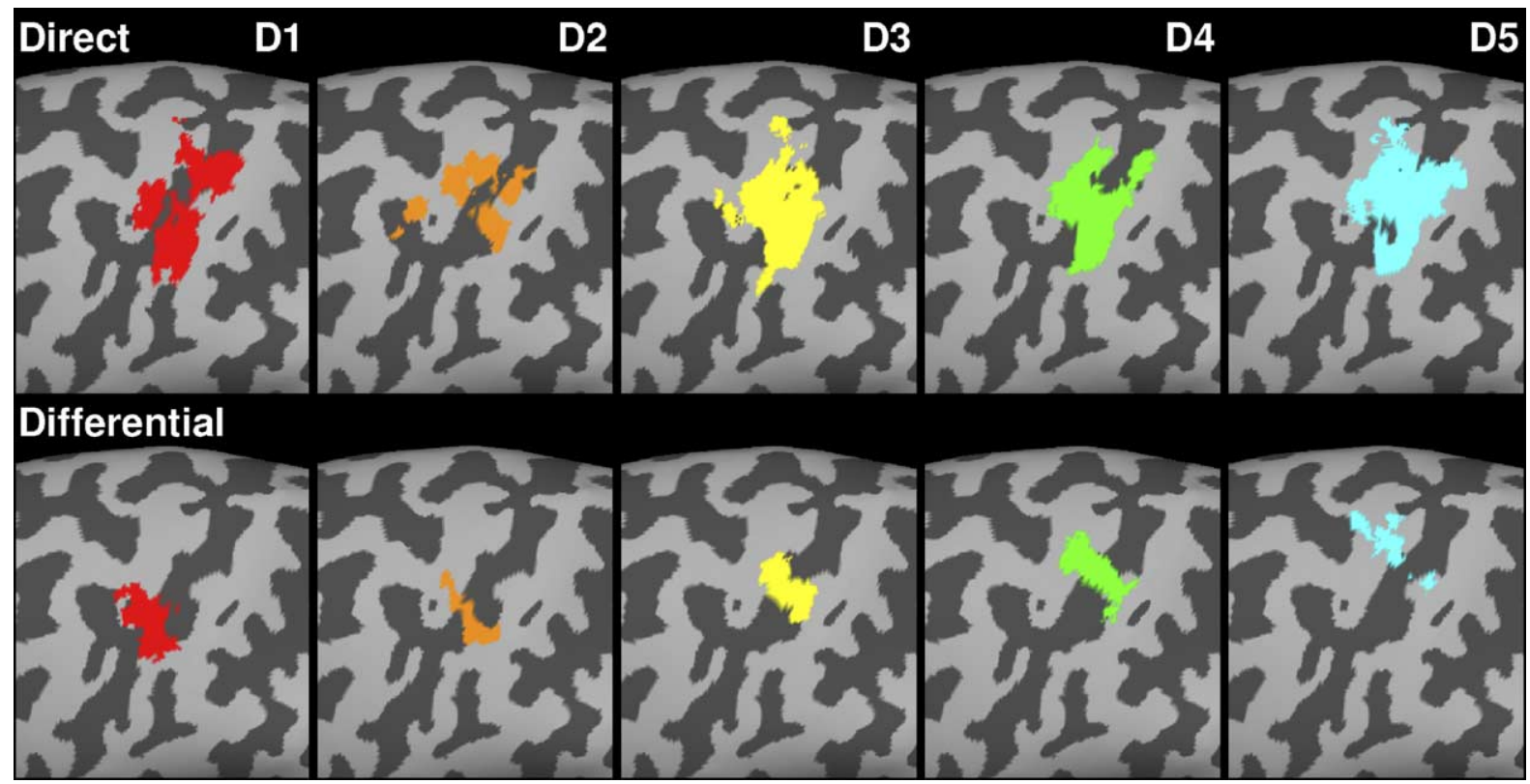

Fig. 23. Functional somatotopy of finger representations in human primary motor cortex. (Top) Whereas direct mapping of individual finger movements vs motor rest results in activation areas with considerable overlap, (bottom) differential mapping of a single finger movement vs movement of another finger yields more segregated and somatotopically arranged cortical representations. The maps for digit D1 (thumb) to D5 (little finger) are reconstructed from multi-slice acquisitions and projected onto the inflated cortex covering the left-hemispheric M1 hand area (single subject). Reprinted from Ref. [121] with permission.

noninvasively mapping human brain function at high spatiotemporal resolution leads to growing expectations in basic and clinical neuroscience. The increasing availability of suitable MRI instruments expands the list of foreseeable applications to the functional diagnosis of neurological and psychiatric dysfunction and the monitoring of cortical reorganization and neurorehabilitation. Technical demands originate from real-time variants of functional MRI which will serve as new means for providing a brain-computer interface ultimately aiming at studies of biofeedback.

In this context it is fair to acknowledge that beyond the methodological difficulties and limitations discussed in this article, the most serious threat to a sustainable development of functional MRI into a robust and reproducible tool for human brain mapping is its uncritical use as a 'camera'. Accordingly, recommendations for sound experimental work mainly refer to a persistent control of the original image quality and a detailed understanding of the various steps taken during data acquisition and data analysis, in particular when using (commercially available) 'black box' programs. In general, it seems advisable to bring together expertise from MRI physics, physiology, and neuropsychology in order to achieve the common purpose of a better understanding of the human brain.

\section{Annex: common BOLD MRI sequences}

As briefly discussed in Section 2.3.1, the most frequently used MRI sequence for functional brain mapping is single-shot EPI. Fig. 24 schematically sketches the EPI

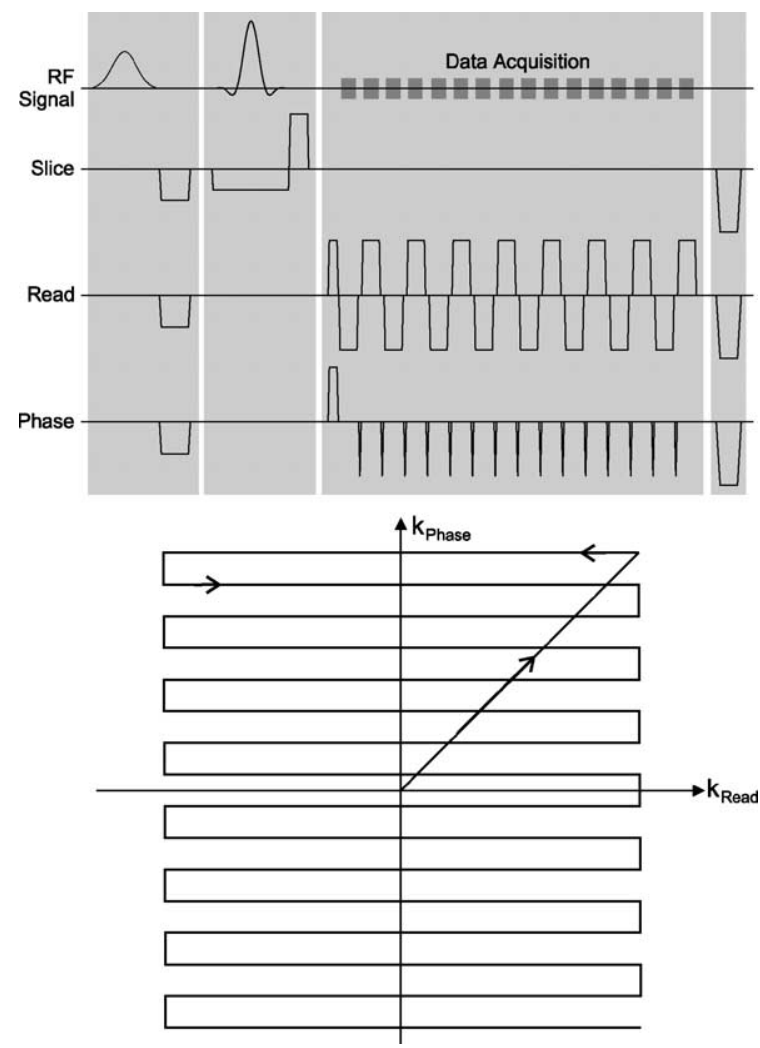

Fig. 24. Echo-planar imaging (EPI). (Top) Schematic timing diagram of the radiofrequency and gradient pulses and (bottom) corresponding trajectory in $k$-space. The example refers to the acquisition of 16 Fourier lines. 
timing diagram of radiofrequency (RF) and gradient pulses together with the corresponding trajectory in $k$-space. A single slice-selective RF excitation pulse and a subsequent acquisition period, which typically comprises 64-128 gradient echoes with alternating sign, characterize the sequence. Apart from this central core, EPI starts with a fat suppression module, that is, a chemical-shift-selective RF excitation of the lipid methylene (and methyl) protons followed by spoiler gradients, and ends with a series of spoiler gradients to remove residual transverse magnetizations before subsequent excitations. Although different waveforms are possible for frequency encoding, most EPI sequences employ trapezoidal read gradients with the actual data acquisition confined to the corresponding flat top periods. Cumulative phase encoding is accomplished by using short gradient 'blips' optimized for maximum slew rate in between successive gradient echoes. Typically, data acquisition starts at a corner of $k$-space so that the low spatial frequencies near the center of $k$-space are acquired in the middle part of the acquisition period at an intermediate echo time.

In principle, EPI sequences present with a number of technical challenges. First of all, the alternating sign of successive $k$-space acquisitions (gradient echoes) may lead to ghosting artifacts after image reconstruction. This problem is usually dealt with by a phase correction using data from initial gradient echoes without phase encoding (not shown). Secondly, MRI signal contributions from chemically shifted compounds such as lipid protons have to be eliminated by fat suppression techniques in order to avoid severe image artifacts. Thirdly, residual resonance offset effects due to magnetic field inhomogeneities or tissue susceptibility differences are in many cases unavoidable and thus lead to focal signal losses and geometric distortions. Moreover, even the "normal' decay of the echo train with the effective spin-spin relaxation time $\mathrm{T} 2 *$ limits the achievable number of gradient echoes, that is, the spatial resolution. This particularly applies to human studies because the maximum slew rate for gradient switching is restricted to avoid peripheral nerve stimulation. And finally, the differential $\mathrm{T} 2{ }^{*}$ attenuation of the acquired Fourier lines broadens the point-spread function of the EPI reconstruction which degrades the nominal resolution by a certain degree of image blurring. On the other hand, the strong potential of single-shot EPI is its speed which allows for a whole-brain coverage at moderate resolution but within repetition times of only $2-3 \mathrm{~s}$.

An alternative to EPI is spiral imaging as demonstrated in Fig. 25. Similar to EPI, the sequence is encompassed by a fat suppression module and a final set of gradient spoilers. Two sinusoidal and cosinusoidal gradients follow the single RF excitation pulse, respectively, which increase in amplitude and decrease in frequency. The scheme results in a spiral trajectory in $k$-space with constant linear velocity. Because this trajectory usually starts at the center of $k$-space, the effective gradient echo time of a spiral sequence

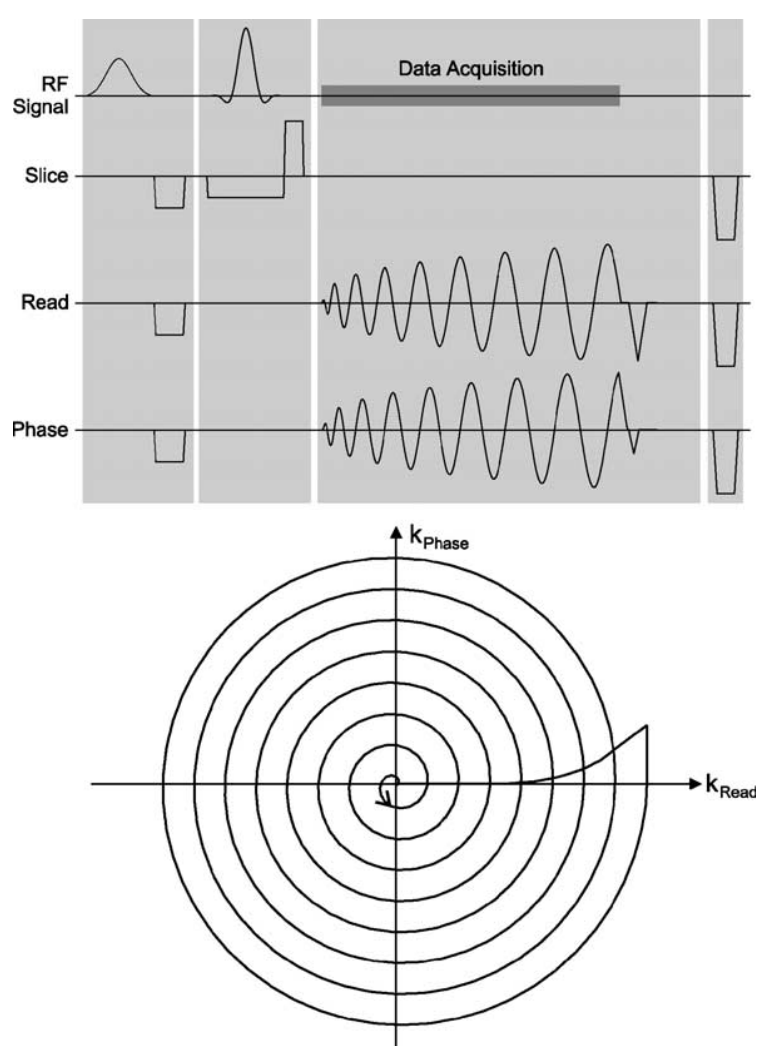

Fig. 25. Spiral imaging. (Top) Schematic timing diagram of the radiofrequency and gradient pulses and (bottom) corresponding trajectory in $k$-space. The example refers to an acquisition which is equivalent to 16 Fourier lines.

is shorter than that of a corresponding EPI sequence. If spiral sequences are not used for BOLD MRI, which of course relies on a prolonged echo time to ensure sufficient $\mathrm{T}^{*}$ contrast, this feature allows for a reduction of phase-related distortions such as flow and motion artifacts. The reconstruction of a spiral image requires a re-gridding of data samples in $k$-space before application of a $2 \mathrm{D}$ Fourier transformation. Noteworthy, the presence of residual off-resonance effects poses a serious problem for spiral imaging. Respective artifacts translate into a radial smearing of intensities and are even worse than observed for EPI. Although these problems are the subject of numerous theoretical and practical studies, no definite solution for practical imaging purposes has yet been found.

When aiming at BOLD MRI with reduced susceptibility artifacts or increased spatial resolution, a possible solution for EPI or spiral imaging sequences is to use multi-shot versions with segmented (partial) $k$-space coverage. Alternatively, high-quality maps at high resolution are even better obtained with use of multi-echo FLASH sequences. Fig. 26 demonstrates that such approaches bear the advantage of scanning $k$-space always along the same direction. This conventional frequency-encoding technique avoids the generation of ghosting artifacts that result from the use of positive and negative read gradients with slightly different gradient integrals at equivalent time points. 


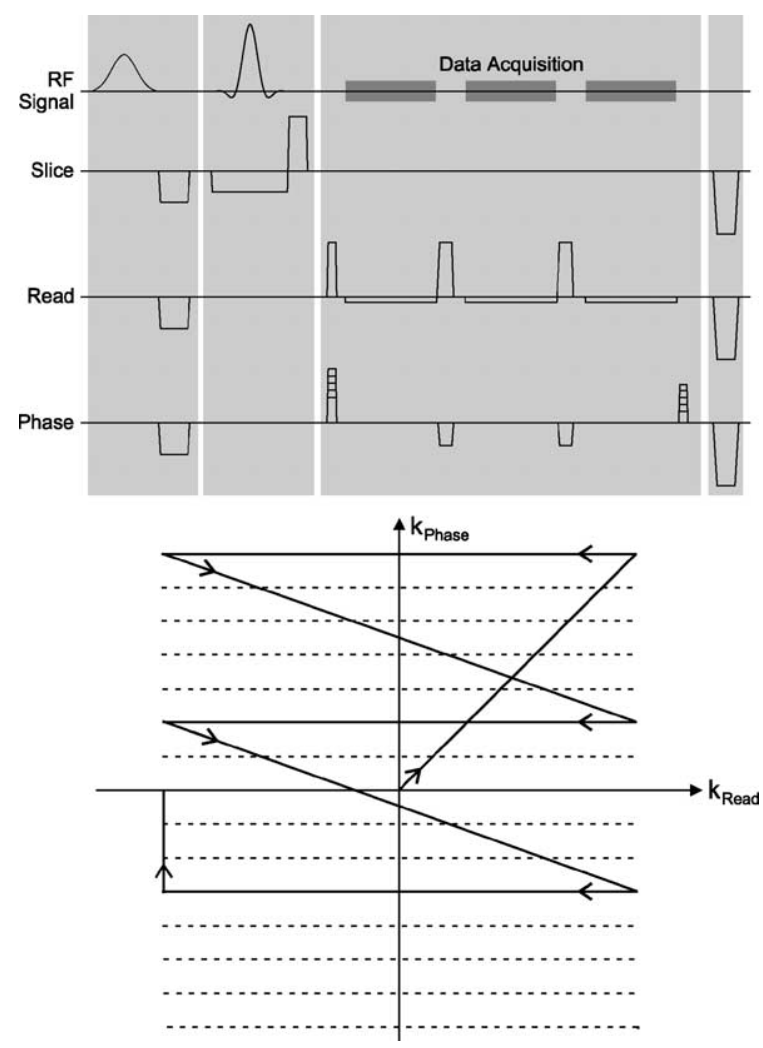

Fig. 26. Multi-echo FLASH imaging. (Top) Schematic timing diagram of the radiofrequency and gradient pulses and (bottom) corresponding trajectory in $k$-space. The example refers to the acquisition of three successive gradient echoes (same sign) representing Fourier lines from three different segments in $k$-space.

Putative image artifacts from minor temporal instabilities of gradient-echo packages from successive repetition intervals may be eliminated by echo-train shifting before on-line image reconstruction [125]. In general, multi-echo FLASH allows for a significantly lower receiver bandwidth than EPI and therefore gains substantial SNR for functional mapping at very high resolution. Recent implementations in our laboratory successfully employed seven echoes with a bandwidth of $217 \mathrm{~Hz} /$ pixel and a mean echo time of $36 \mathrm{~ms}$ (at a field strength of $3 \mathrm{~T}$ ). A matrix of $259 \times 256$ data points together with a $128 \times 128 \mathrm{~mm}^{2}$ field-of-view led to an in-plane resolution of $0.5 \mathrm{~mm}$ (without interpolation) and a repetition time of $3 \mathrm{~s}$ (including fat suppression).

\section{References}

[1] M.E. Moseley, G.H. Glover, Clin. N. Am. 5 (1995) 161.

[2] P.A. Bandettini, E.C. Wong, Clin. N. Am. 8 (1997) 345.

[3] K. Uğurbil, X. Hu, W. Chen, X.H. Zhu, S.G. Kim, A. Georgopoulos, Philos. Trans. R. Soc. Lond. B 354 (1999) 1195.

[4] J. Frahm, P. Fransson, G. Krüger, in: U. Windhorst, H. Johansson (Eds.), Modern Techniques in Neuroscience Research, Springer, Berlin, 1999, p. 1055.

[5] J.A. Detre, T.F. Floyd, Neuroscientist 7 (2001) 64.

[6] R.S. Menon, Curr. Opin. Neurobiol. 11 (2001) 630.

[7] S.G. Kim, S. Ogawa, Curr. Opin. Neurobiol. 12 (2002) 607.
[8] S. Ogawa, T.M. Lee, A.R. Kay, D.W. Tank, Proc. Natl. Acad. Sci. USA 87 (1990) 9868.

[9] K.K. Kwong, J.W. Belliveau, D.A. Chesler, I.E. Goldberg, R.M Weisskoff, B.P. Poncelet, D.N. Kennedy, B.E. Hoppel, M.S. Cohen, R. Turner, H.M. Cheng, T.J. Brady, B.R. Rosen, Proc. Natl. Acad. Sci. USA 89 (1992) 5675.

[10] P.A. Bandettini, E.C. Wong, R.S. Hinks, R.S. Tikofsky, J.S. Hyde, Magn. Reson. Med. 25 (1992) 390.

[11] J. Frahm, H. Bruhn, K.D. Merboldt, W. Hänicke, J. Magn. Reson. Imaging 2 (1992) 501.

[12] A.M. Blamire, S. Ogawa, K. Uğurbil, D. Rothman, G. McCarthy, J.M. Ellermann, F. Hyder, Z. Rattner, R.G. Shulman, Proc. Natl. Acad. Sci. USA 89 (1992) 11069.

[13] J.W. Belliveau, D.N. Kennedy, R.C. McKinstry, B.R. Buchbinder, R.M. Weisskoff, M.S. Cohen, J.M. Vevea, T.J. Brady, B.R. Rosen, Science 254 (1991) 716.

[14] J.A. Detre, J.S. Leigh, D.S. Williams, A.P. Koretsky, Magn. Reson. Med. 23 (1992) 37.

[15] J.A. Detre, D.C. Alsop, Eur. J. Radiol. 30 (1999) 115.

[16] J.A. Detre, J. Wang, Clin. Neurophysiol. 113 (2002) 621.

[17] E.T. Ahrens, P.T. Narasimhan, T. Nakada, R.E. Jacobs, Prog. NMR Spectrosc. 40 (2002) 275.

[18] Y.J. Lin, A.P. Koretsky, Magn. Reson. Med. 38 (1997) 378.

[19] R.G. Pautler, A.C. Silva, A.P. Koretsky, Magn. Reson. Med. 40 (1998) 740.

[20] T.Q. Duong, A.C. Silva, A.P. Lee, S.G. Kim, Magn. Reson. Med. 43 (2000) 383.

[21] T. Watanabe, T. Michaelis, J. Frahm, Magn. Reson. Med. 46 (2001) 424.

[22] T. Watanabe, O. Natt, S. Boretius, J. Frahm, T. Michaelis, Magn. Reson. Med. 48 (2002) 852.

[23] K.S. Saleem, J.M. Pauls, M. Augath, T. Trinath, B.A. Prause, T. Hashikawa, N.K. Logothetis, Neuron 34 (2002) 685.

[24] R.G. Pautler, R. Mongeau, R.E. Jacobs, Magn. Reson. Med. 50 (2003) 33.

[25] T. Watanabe, J. Radulovic, J. Spiess, O. Natt, S. Boretius, J. Frahm, T. Michaelis, Neuroimage 00 (2004) 000.

[26] M. Hoehn, E. Küstermann, J. Blunk, D. Wiedermann, T. Trapp, S. Wecker, M. Föcking, H. Arnold, J. Hecheler, B.K. Fleichsmann, W. Schwindt, C. Bührle, Proc. Natl. Acad. Sci. USA 99 (2002) 16267.

[27] J.W. Bulte, I.D. Duncan, J.A. Frank, J. Cereb. Blood Flow Metab. 22 (2002) 899.

[28] D. Shahbazi-Gahrouei, M. Williams, S. Rizvi, B.J. Allen, J. Magn. Reson. Imaging 14 (2001) 169.

[29] D. Artemov, N. Mori, B. Okollie, Z.M. Bhujwala, Magn. Reson. Med. 49 (2003) 403.

[30] A. Moore, J.P. Basilion, E.A. Chiocca, R. Weissleder, Biochim. Biophys. Acta 1402 (1998) 239.

[31] A.Y. Louie, M.M. Hüber, E.T. Ahrens, U. Rothbächer, R. Moats, R.E. Jacobs, S.E. Fraser, T.J. Meade, Nature Biotechnol. 18 (2000) 321.

[32] R. Weissleder, A. Moore, U. Mahmood, R. Bhorade, H. Benveniste, E.A. Chiocca, J.P. Basilion, Nature Med. 6 (2000) 351.

[33] D.C. Sullivan, J.M. Hoffman, Semin. Radiat. Oncol. 11 (2001) 37.

[34] C. Bremer, R. Weissleder, Acad. Radiol. 8 (2001) 15.

[35] C.D. Binnie, P.F. Prior, J. Neurol. Neurosurg. Psychiatry 57 (1994) 1308.

[36] C.M. Michel, G. Thut, S. Morand, A. Khateb, A.J. Pegna, R. Grave de Peralta, S. Gonzalez, M. Seeck, T. Landis, Brain Res. Brain Res. Rev. 36 (2001) 108.

[37] R. Hari, Electroencephalogr. Clin. Neurophysiol. Suppl. 47 (1996) 47.

[38] A. Villringer, U. Dirnagl, Cerebrovasc. Brain Metab. Rev. 7 (1995) 240.

[39] R.B. Buxton, L.R. Frank, J. Cereb. Blood Flow Metab. 17 (1997) 64

[40] R.B. Buxton, E.C. Wong, L.R. Frank, Magn. Reson. Med. 39 (1998) 855. 
[41] J.B. Mandeville, J.J. Marota, C. Ayata, G. Zaharchuk, M.A. Moskowitz, B.R. Rosen, R.M. Weisskoff, J. Cereb. Blood Flow Metab. 19 (1999) 679.

[42] R.D. Hoge, J. Atkinson, B. Gill, G.R. Crelier, S. Marrett, G.B. Pike, Magn. Reson. Med. 42 (1999) 849.

[43] R.D. Hoge, G.B. Pike, J. Chem. Neuroanat. 22 (2001) 43.

[44] K.J. Friston, A. Mechelli, R. Turner, C.J. Price, Neuroimage 12 (2000) 466.

[45] Y. Zheng, J. Martindale, D. Johnston, M. Jones, J. Berwick, J. Mayhew, Neuroimage 16 (2002) 617

[46] Y. Yang, G.H. Glover, P. van Gelderen, A.C. Patel, V.S. Mattay, J.A. Frank, J.H. Duyn, Magn. Reson. Med. 39 (1998) 61 Erratum: Magn. Reson. Med. 39 (1998) 505.

[47] W. Irnich, F. Schmitt, Magn. Reson. Med. 33 (1995) 619.

[48] M. Brand, O. Heid, Magn. Reson. Med. 48 (2002) 731.

[49] J.A. Den Boer, J.D. Bourland, J.A. Nyenhuis, C.L. Ham, J.M. Engels, F.X. Hebrank, G. Frese, D.J. Schaefer, J. Magn. Reson. Imaging 15 (2002) 520.

[50] F. Liu, H. Zhao, S. Crozier, IEEE Trans. Biomed. Eng. 50 (2003) 804.

[51] B.A. Chronik, M. Ramachandran, J. Magn. Reson. Imaging 17 (2003) 716.

[52] R.S. Menon, C.G. Thomas, J.S. Gati, NMR Biomed. 10 (1997) 179.

[53] F.G. Hoogenraad, P.J. Pouwels, M.B. Hofman, S.A. Rombouts, C. Lavini, M.O. Leach, E.M. Haacke, Magn. Reson. Imaging 18 (2000) 405

[54] G.H. Glover, S. Lai, Magn. Reson. Med. 39 (1998) 361.

[55] D.K. Sodickson, W.J. Manning, Magn. Reson. Med. 38 (1997) 591.

[56] K.P. Pruessmann, M. Weiger, M.B. Scheidegger, P. Boesiger, Magn. Reson. Med. 42 (1999) 952.

[57] D.K. Sodickson, C.A. McKenzie, Med. Phys. 28 (2001) 1629.

[58] M.A. Griswold, P.M. Jakob, R.M. Heidemann, M. Nittka, V. Jellus, J. Wang, B. Kiefer, A. Haase, Magn. Reson. Med. 47 (2002) 1202.

[59] T.Q. Duong, E. Yacoub, G. Adriany, X. Hu, K. Uğurbil, J.T. Vaughan, H. Merkle, S.G. Kim, Magn. Reson. Med. 48 (2002) 589.

[60] J.L. Boxerman, P.A. Bandettini, K.K. Kwong, J.R. Baker, T.L. Davis, B.R. Rosen, R.M. Weisskoff, Magn. Reson. Med. 34 (1995) 4.

[61] J.L. Boxerman, L.M. Hamberg, B.R. Rosen, R.M. Weisskoff, Magn. Reson. Med. 34 (1995) 555.

[62] N. Fujita, Magn. Reson. Med. 46 (2001) 723.

[63] D. Malonek, A. Grinvald, Science 272 (1996) 551.

[64] P. Fransson, G. Krüger, K.D. Merboldt, J. Frahm, Magn. Reson. Med. 39 (1998) 912

[65] P.A. Bandettini, A. Jesmanowicz, E.C. Wong, J.S. Hyde, Magn. Reson. Med. 30 (1993) 161.

[66] J. Baudewig, P. Dechent, K.D. Merboldt, J. Frahm, Magn. Reson. Imaging 21 (2003) 1121.

[67] A. Kleinschmidt, M. Requardt, K.D. Merboldt, J. Frahm, Int. J. Imaging Syst. Technol. 6 (1995) 238.

[68] P. Fransson, G. Krüger, K.D. Merboldt, J. Frahm, Neuroimage 9 (1999) 611.

[69] P. Dechent, J. Frahm, Brain Res. Cogn. Brain Res. (2004) in press.

[70] M. Lauritzen, L. Gold, J. Neurosci. 23 (2003) 3972

[71] N.K. Logothetis, J. Neurosci. 23 (2003) 3963.

[72] N.K. Logothetis, J. Pauls, M. Augath, T. Trinath, A. Oeltermann, Nature 412 (2001) 150.

[73] C. Mathiesen, K. Caesar, N. Akgoren, M. Lauritzen, J. Physiol. 512 (1998) 555.

[74] N.K. Logothetis, Philos. Trans. R. Soc. Lond. B 357 (2002) 1003.

[75] D.E. Bohning, A. Shastri, K.A. McConnell, Z. Nahas, J.P. Lorberbaum, D.R. Roberts, C. Teneback, D.J. Vincent, M.S. George, Biol. Psychiatry 45 (1999) 385.

[76] A. Shastri, M.S. George, D.E. Bohning, Electroencephalogr. Clin. Neurophysiol. Suppl. 51 (1999) 55.

[77] J.C. Rothwell, J. Neurosci. Meth. 27 (1997) 113.

[78] J. Baudewig, H.R. Siebner, S. Bestmann, F. Tergau, T. Tings, W. Paulus, J. Frahm, Neuroreport 12 (2001) 3543.
[79] J. Spiegel, J. Tintera, J. Gawehn, P. Stoeter, R.D. Treede, Clin. Neurophysiol. 110 (1999) 47.

[80] S. Bestmann, J. Baudewig, J. Frahm, J. Magn. Reson. Imaging 17 (2003) 309.

[81] S. Bestmann, J. Baudewig, H.R. Siebner, J.C. Rothwell, J. Frahm, Neuroimage 20 (2003) 1685.

[82] J. Baudewig, M.A. Nitsche, W. Paulus, J. Frahm, Magn. Reson. Med. 45 (2001) 196.

[83] J. Frahm, K.D. Merboldt, W. Hänicke, Magn. Reson. Med. 29 (1993) 139

[84] R.S. Menon, B.G. Goodyear, Magn. Reson. Med. 41 (1999) 230.

[85] P. Dechent, J. Frahm, Neuroreport 11 (2000) 3247.

[86] K. Cheng, R.A. Waggoner, K. Tanaka, Neuron 32 (2001) 359.

[87] B.G. Goodyear, R.S. Menon, Hum. Brain Mapp. 14 (2001) 210.

[88] J.C. Horton, E.T. Hedley-Whyte, Philos. Trans. R. Soc. Lond. B 304 (1984) 255.

[89] P. Fransson, K.D. Merboldt, M. Ingvar, K.M. Petersen, J. Frahm, Neuroreport 12 (2001) 1415.

[90] P. Fransson, K.D. Merboldt, K.M. Petersen, M. Ingvar, J. Frahm, Neuroimage 16 (2002) 977.

[91] K.J. Friston, A.P. Holmes, K.P. Worsley, J.B. Poline, C.D. Frith, R.S.J. Frackowiak, Hum. Brain Mapp. 2 (1995) 189.

[92] P. Fransson, G. Krüger, K.D. Merboldt, J. Frahm, Neuroreport 9 (1998) 2001

[93] P. Fransson, G. Krüger, K.D. Merboldt, J. Frahm, Magn. Reson. Imaging 17 (1999) 1

[94] B. Biswal, F.Z. Yetkin, V.M. Haughton, J.S. Hyde, Magn. Reson. Med. 34 (1995) 537.

[95] A. Salek-Haddadi, K.J. Friston, L. Lemieux, D.R. Fish, Brain Res. Brain Res. Rev. 43 (2003) 110.

[96] H. Laufs, K. Krakow, P. Sterzer, E. Eger, A. Beyerle, A. SalekHaddadi, A. Kleinschmidt, Proc. Natl. Acad. Sci. USA 100 (2003) 11053.

[97] J. Baudewig, H.J. Bittermann, W. Paulus, J. Frahm, Clin. Neurophysiol. 112 (2001) 1196.

[98] L. Lemieux, A. Salek-Haddadi, O. Josephs, P. Allen, N. Toms, C. Scott, K. Krakow, R. Turner, D.R. Fish, Neuroimage 14 (2001) 780 .

[99] A. Salek-Haddadi, M. Merschhemke, L. Lemieux, D.R. Fish, Neuroimage 16 (2002) 32.

[100] A. Salek-Haddadi, L. Lemieux, M. Merschhemke, K.J. Friston, J.S. Duncan, D.R. Fish, Ann. Neurol. 53 (2003) 663.

[101] A. Al-Asmi, C.G. Benar, D.W. Gross, Y.A. Khani, F. Andermann, B. Pike, F. Dubeau, J. Gotman, Epilepsia 44 (2003) 1328.

[102] S. Boor, G. Vucurevic, C. Pfleiderer, P. Stoeter, G. Kutschke, R. Boor, Epilepsia 44 (2003) 688.

[103] J. Frahm, K.D. Merboldt, W. Hänicke, A. Kleinschmidt, H. Boecker, NMR Biomed. 7 (1994) 45.

[104] J.V. Hajnal, R. Myers, A. Oatridge, J.E. Schwieso, I.R. Young, G.M. Bydder, Magn. Reson. Med. 31 (1994) 283.

[105] J. Frahm, K.D. Merboldt, W. Hänicke, J. Magn. Reson. B 109 (1995) 234

[106] K.D. Merboldt, J. Finsterbusch, J. Frahm, J. Magn. Reson. 145 (2000) 184

[107] K.D. Merboldt, P. Fransson, H. Bruhn, J. Frahm, Neuroimage 14 (2001) 253.

[108] E. Martin, T. Thiel, P. Joeri, T. Loenneker, D. Ekatodramis, T. Huisman, J. Hennig, V.L. Marcar, Hum. Brain Mapp. 10 (2000) 132.

[109] A.P. Born, I. Law, T.E. Lund, E. Rostrup, L.G. Hanson, G. Wildschiodtz, H.C. Lou, O.B. Paulson, Neuroimage 17 (2002) 1325

[110] E. Martin, V.L. Marcar, Magn. Reson. Imaging Clin. N. Am. 9 (2001) 231.

[111] M.H. Ross, D.A. Yurgelun-Todd, P.F. Renshaw, L.C. Maas, J.H Mendelson, N.K. Mello, B.M. Cohen, J.M. Levin, Neurology 48 (1997) 173. 
[112] V. Hesselmann, O. Zaro Weber, C. Wedekind, T. Krings, O. Schulte, H Kugel, B. Krug, N. Klug, K.J. Lackner, Neurosci. Lett. 308 (2001) 141.

[113] A. Kleinschmidt, H. Bruhn, G. Krüger, K.D. Merboldt, G. Stoppe, J. Frahm, NMR Biomed. 12 (1999) 286.

[114] H. Bruhn, A. Kleinschmidt, H. Boecker, K.D. Merboldt, W Hänicke, J. Frahm, J. Cereb. Blood Flow Metab. 14 (1994) 742.

[115] A. Kleinschmidt, H. Bruhn, H. Steinmetz, J. Frahm, J. Cereb. Blood Flow Metab. 15 (1995) S527.

[116] H. Bruhn, P. Fransson, J. Frahm, J. Magn. Reson. Imaging 13 (2001) 325 .

[117] A. Kleinschmidt, H. Steinmetz, M. Sitzer, K.D. Merboldt, J. Frahm, Stroke 26 (1995) 106.
[118] S. Vorstrup, L. Henriksen, O.B. Paulson, J. Clin. Invest. 74 (1984) 1634

[119] S. Treue, Curr. Opin. Neurobiol. 13 (2003) 428.

[120] K.D. Merboldt, J. Baudewig, S. Treue, J. Frahm, Neuroreport 13 (2002) 1721

[121] P. Dechent, J. Frahm, Hum. Brain Mapp. 18 (2003) 272.

[122] J.N. Sanes, J.P. Donoghue, V. Thangaraj, R.R. Edelman, S. Warach, Science 268 (1995) 1775.

[123] A. Kleinschmidt, M.F. Nitschke, J. Frahm, Eur. J. Neurosci. 9 (1997) 2178

[124] M.H. Schieber, J. Neurophysiol. 86 (2001) 2125

[125] D.A. Feinberg, K. Oshio, J. Magn. Reson. 97 (1992) 177 